\title{
A TIDAL DISRUPTION EVENT IN A NEARBY GALAXY HOSTING AN INTERMEDIATE MASS BLACK HOLE
}

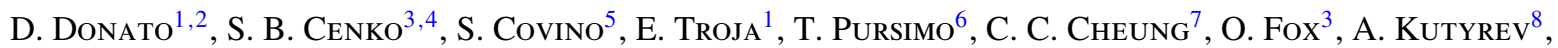 \\ S. CAMPANA ${ }^{4}$, D. FugazzA ${ }^{4}$, H. LANDT $^{9}$, AND N. R. ButLER ${ }^{10}$ \\ ${ }^{1}$ CRESST and Astroparticle Physics Laboratory NASA/GSFC, Greenbelt, MD 20771, USA; davide.donato-1@ nasa.gov \\ 2 Department of Astronomy, University of Maryland, College Park, MD 20742, USA \\ ${ }^{3}$ Astrophysics Science Division, NASA/GSFC, Mail Code 661, Greenbelt, MD 20771, USA \\ ${ }^{4}$ Joint Space Science Institute, University of Maryland, College Park, MD 20742, USA \\ ${ }^{5}$ INAF, Osservatorio Astronomico di Brera, via E. Bianchi 46, I-23807 Merate (LC), Italy \\ ${ }^{6}$ Nordic Optical Telescope, Apartado 474, E-38700 Santa Cruz de La Palma, Spain \\ ${ }^{7}$ Space Science Division, Naval Research Laboratory, Washington, DC 20375-5352, USA \\ 8 Observational Cosmology Laboratory, NASA/GSFC, 8800 Greenbelt Road, Greenbelt, MD 20771-2400, USA \\ ${ }^{9}$ Department of Physics, Durham University, South Road, Durham DH1 3LE, UK \\ ${ }^{10}$ School of Earth and Space Exploration, Arizona State University, Tempe, AZ 85287, USA \\ Received 2013 July 26; accepted 2013 November 22; published 2014 January 8
}

\begin{abstract}
We report the serendipitous discovery of a bright point source flare in the Abell cluster A1795 with archival EUVE and Chandra observations. Assuming the EUVE emission is associated with the Chandra source, the X-ray 0.5-7 keV flux declined by a factor of 2300 over a time span of $6 \mathrm{yr}$, following a power-law decay with index $\sim 2.44 \pm 0.40$. The Chandra data alone vary by a factor of $\sim 20$. The spectrum is well fit by a blackbody with a constant temperature of $k T \sim 0.09 \mathrm{keV}\left(\sim 10^{6} \mathrm{~K}\right)$. The flare is spatially coincident with the nuclear region of a faint, inactive galaxy with a photometric redshift consistent at the $1 \sigma$ level with the cluster $(z=0.062476)$. We argue that these properties are indicative of a tidal disruption of a star by a black hole $(\mathrm{BH})$ with $\log \left(M_{\mathrm{BH}} / M_{\odot}\right) \sim 5.5 \pm 0.5$. If so, such a discovery indicates that tidal disruption flares may be used to probe BHs in the intermediate mass range, which are very difficult to study by other means.
\end{abstract}

Key words: galaxies: clusters: individual (A1795) - galaxies: general - galaxies: nuclei - X-rays: galaxies

Online-only material: color figures

\section{INTRODUCTION}

There now exists compelling evidence that most, if not all, massive bulge galaxies harbor supermassive black holes (SMBH, $M_{\mathrm{BH}} \geqslant 10^{6} M_{\odot}$ ) in their nuclei (e.g., Kormendy \& Richstone 1995). During their lifetime those galaxies can experience a phase of high activity when the gas is rapidly falling into the black hole $(\mathrm{BH})$ through an accretion disk (e.g., Hopkins $\&$ Hernquist 2006). But even in the subsequent quiescent phase, the BH can be fed by stars whose orbits are too close to escape its large gravitational potential well (Frank \& Rees 1976). Such tidal disruption events (TDEs) can lead to bright X-ray/UV flares as a fraction of the disrupted material accretes onto the BH (Rees 1988). In addition to the X-ray/UV emission from the disk, radio emission has now been detected in several TDE candidates. This is interpreted as synchrotron emission from electrons accelerated by a relativistic jet formed during the disruption process (Bloom et al. 2011; Zauderer et al. 2011). Indeed, only a few cases of a TDE accompanied by the birth of the relativistic jet have been discovered. In GRB 110328A/Sw J1644+57 (Burrows et al. 2011; Levan et al. 2011; Zauderer et al. 2011) and Sw J2058+05 (Cenko et al. 2012) the radio emission has been observed simultaneously to the X-ray discovery with the Swift satellite, while a radio source has been observed both in the nucleus of the galaxy IC 3599 and at the position of the transient RX J1420.4+5334 nine and $22 \mathrm{yr}$ after the initial $\mathrm{X}$-ray detection, respectively (Bower et al. 2013 and references therein).

Before these Swift discoveries, the first TDE candidates were identified in archival X-ray data, either in the Röntgensatellit all-sky survey (ROSAT; Grupe et al. 1995; Bade et al. 1996; Komossa \& Greiner 1999; Greiner et al. 2000; Donley et al. 2002; Cappelluti et al. 2009), in the X-ray Multi-Mirror slew survey (XMM-Newton; Esquej et al. 2008, 2010), or in observed fields by XMM-Newton and Chandra (Komossa et al. 2004; Maksym et al. 2010; Lin et al. 2011; Saxton et al. 2012). More recently, other candidates have been found as a result of ongoing, real-time surveys. Some examples include transients discovered by the Galaxy Evolution Explorer (GALEX; Gezari et al. 2006, 2008, 2009), in the Sloan Digital Sky Survey (SDSS; Komossa et al. 2008; van Velzen et al. 2011), in the PanSTARRS1 Medium Deep Survey (Gezari et al. 2012; Chornock et al. 2013), and by the Palomar Transient Factory (Cenko et al. 2012).

In almost all these cases, the event occurred in a normal galaxy (i.e., without Seyfert activity); the flaring source reached an X-ray/UV luminosity of $L>10^{42} \mathrm{erg} \mathrm{s}^{-1}$ and faded by at least one to two orders of magnitude on timescales of months/years; and, the spectral energy distribution (SED) was characterized by a blackbody (BB) with a temperature $T \gtrsim 10^{5} \mathrm{~K}$, as expected from an accretion disk (Komossa \& Greiner 1999; Esquej et al. 2007; Cappelluti et al. 2009; Maksym et al. 2010). It has been argued (Rees 1988; Evans \& Kochanek 1989; Phinney 1989) that the mass accretion rate should follow a $\sim t^{-n}$ power-law where $n=5 / 3$; however, more recent analytic works and hydrodynamical simulations (Strubbe \& Quataert 2009, 2011; Lodato et al. 2009; Lodato \& Rossi 2011; Guillochon \& Ramirez-Ruiz 2013) suggest that the flux in any given band may deviate from this simple power-law. At early times the slope is expected to be flatter while at later times the slope asymptotes to $\sim 2.2$ for approximately half of the stellar disruptions. Guillochon \& Ramirez-Ruiz (2013) consider the possibility that the star is only partially disrupted, with the stellar core surviving the encounter and with the stellar outer gas becoming bound to the $\mathrm{BH}$. In all of these cases, the index is steeper than $n=5 / 3$. 
Observationally, the typical X-ray light curve is poorly sampled and the slope of decline is consequently not well constrained. In a few cases when the event is extensively monitored (e.g., Sw J2058+05, Cenko et al. 2012) or is detected by chance on multiple occasions (Cappelluti et al. 2009), a steep decline with $n \sim 2.2$ is observed. The two Swift discoveries (Sw J1644+57 and Sw J2058+05) show also significant variation on relatively short timescales (minutes to days). On timescales of a few hours, the X-ray flux of these two transients changed by a factor of 100 and 1.5 , respectively.

The hosts in nearly all the studied cases have been either a quiescent or a star-forming galaxy. Morphologically they range from being ellipticals/S0 to spirals with, typically, an evident bulge. The common feature among the hosts is the estimated $\mathrm{BH}$ mass: regardless of the luminosity scaling relation used to infer it, the $\mathrm{BH}$ is thought to be supermassive $\left(M_{\mathrm{BH}} / M_{\odot} \sim 10^{6}-10^{7}\right)$. Only in a few cases an intermediate mass BH (IMBH, $10^{2} \lesssim$ $M_{\mathrm{BH}} / M_{\odot} \lesssim 10^{6}$ ) has been proposed by some authors. However, all of these cases differ from previous traditional TDEs because the disrupted object is not a main sequence star: a white dwarf for Swift J1644+57 (Krolik \& Piran 2011), the gamma-ray bursts GRB 060218 (Shcherbakov et al. 2012) and GRB 060614 (Lu et al. 2008), a flare in an extragalactic globular cluster (Irwin et al. 2010), and a super-Jupiter object for IGR J12580+0134 (Nikołajuk \& Walter 2013).

In this work we present the serendipitous discovery of an extremely bright point source in archival observations with the Extreme Ultraviolet Explorer (EUVE) and Chandra of the field of the moderately rich (richness class 2) cluster A1795 $(z=0.062476$, Hill \& Oegerle 1993). The large X-ray flux variation (with a light curve characterized by a power-law decay), together with the shape of the Chandra spectra suggests that this is a classical TDE, while the characteristics of the putative host galaxy identified in optical and infrared observations further suggest that the host is harboring an IMBH. In the final stages of preparation of this manuscript, an independent discovery of this source was reported by Maksym et al. (2013). While these authors came to largely similar conclusions regarding the origin of the transient, in this work we present (1) a more detailed light curve analysis based on very recent simulation studies to support our interpretation of the transient nature as a tidal event; (2) stringent limits on the jet emission using extensive archival analysis of Very Large Array (VLA) data; (3) new broadband photometric data and significantly deeper spectroscopic limits that better constrain the characteristics of the host galaxy.

The paper is organized as follows: In Section 2 we present the data reduction and analysis of new and archival observations, in Section 3 we show the evidence supporting the TDE scenario and in Section 4 we discuss the results. A summary is given in Section 5. Throughout the paper, a concordance cosmology with $H_{0}=71 \mathrm{~km} \mathrm{~s}^{-1} \mathrm{Mpc}^{-1}, \Omega_{\Lambda}=0.73$, and $\Omega_{\mathrm{m}}=0.27$ (Spergel et al. 2003) is adopted. Quoted errors are $90 \%$ confidence levels for the X-ray analysis results and $68 \%$ in all the other cases. All the upper limits are at the $3 \sigma$ level unless stated otherwise.

\section{OBSERVATIONS AND DATA ANALYSIS}

Since its launch in 1999, Chandra observed the field of the galaxy cluster A1795 several times. In the first pointing taken in 1999 December, a very bright point-like source, which we refer to hereafter as CXO J1348, was detected 0.7 west of the cD elliptical galaxy located at the center of the cluster. In the following $4 \mathrm{yr}$, Chandra re-observed the same field five times
Table 1

Observation Log of Chandra Pointings through 2005

\begin{tabular}{lcccc}
\hline \hline $\begin{array}{l}\text { ObsID } \\
(1)\end{array}$ & $\begin{array}{c}\text { Date } \\
(2)\end{array}$ & $\begin{array}{c}\text { ACIS } \\
(3)\end{array}$ & $\begin{array}{c}\text { Exp. } \\
(4)\end{array}$ & $\begin{array}{c}c / r \\
(5)\end{array}$ \\
\hline 00494 & 1999 Dec 20 & $\mathrm{S}$ & 19.5 & $10.5 \pm 0.9$ \\
00493 & 2000 Mar 21 & $\mathrm{S}$ & 19.6 & $7.6 \pm 0.7$ \\
03666 & 2002 Jun 10 & $\mathrm{S}$ & 14.4 & $1.9 \pm 0.4$ \\
05287 & 2004 Jan 14 & $\mathrm{S}$ & 14.3 & $<0.5$ \\
05288 & 2004 Jan 16 & $\mathrm{S}$ & 14.6 & $1.4 \pm 0.4$ \\
05289 & 2004 Jan 18 & $\mathrm{I}$ & 15.0 & $1.3 \pm 0.3$ \\
06160 & 2005 Mar 20 & $\mathrm{S}$ & 14.8 & $<0.5$ \\
06162 & 2005 Mar 28 & $\mathrm{I}$ & 13.6 & $<0.6$ \\
06163 & 2005 Mar 31 & $\mathrm{I}$ & 14.9 & $<0.6$ \\
\hline
\end{tabular}

Notes. Column explanations: $1=$ observation ID; 2 =observation date; $3=$ instrument where the position of CXO J1348 is localized; $4=$ exposure time in $\mathrm{ks} ; 5=$ net count rates for detections in units of $10^{-3}$ counts $\mathrm{s}^{-1}$ in the $0.3-8 \mathrm{keV}$ range.

(see Table 1 for a detailed description of the Chandra pointings). In these observations the source is detected but with a declining intensity. From 2005 to 2012, the transient position fell within the Chandra field of view 20 additional times, but no emission was detected at this position.

Intrigued by this behavior, we searched the archives of other satellites and telescopes and in the literature. We found that a few months before the first Chandra observation, a target of opportunity (ToO) was granted by the Advanced Satellite for Cosmology and Astrophysics (ASCA) team to observe a giant outburst from a galaxy in A1795 discovered in archival EUVE data (Bowyer et al. 1999; Bonamente et al. 2001). This outburst appeared for the first time in an EUVE observation performed in 1998 March 27 and disappeared in the following months. Bowyer et al. (1999) reported that the radial profile of the UV emission was consistent with being produced by a point source. The ASCA ToO was triggered in coordination with further $E U V E$ follow-up observations to determine its presence in the $\mathrm{UV} / \mathrm{X}$-rays and its nature. Unfortunately, the large ASCA pointspread function (PSF) and the bright intensity of the galaxy cluster in the X-ray prevented any detection, and no further action was taken. The point source was never detected again by $E U V E$, suggesting a transient origin.

We checked for additional data recorded by other X-ray satellites spanning the 1992-2004 time range. The ROSAT data provide useful timing constraints: four observations were obtained with the High Resolution Imager from 1992 July 25 to 1997 July 23. For all of them we did not detect any X-ray emission at the position of CXO J1348. An upper limit in the $0.1-2.4 \mathrm{keV}$ range for the count rate of the last observation (lasting $8.8 \mathrm{ks}$ ) can be set at $<4.42 \times 10^{-3} \mathrm{ct} \mathrm{s}^{-1}$. Assuming a BB spectrum with $k T=0.09 \mathrm{keV}$ (an average value obtained fitting the Chandra spectra of the observations taken in the 1999-2002 period; see Section 2.1.2) and Galactic absorption $\left(N_{\mathrm{H}, \mathrm{Gal}}=1.19 \times 10^{20} \mathrm{~cm}^{-2}\right.$, Kalberla et al. 2005), we estimate with WebPIMMS an unabsorbed flux upper limit, extrapolated to the $0.5-7 \mathrm{keV}$ range, of $\sim 2.9 \times 10^{-14} \mathrm{erg} \mathrm{cm}^{-2} \mathrm{~s}^{-1}$ for the observation in 1997.

Data from other X-ray satellites, such as XMM-Newton, BeppoSAX, Swift, and Suzaku, were not used for one or more of the following reasons: (1) the satellite has a larger PSF and a much lower sensitivity than Chandra, allowing the cluster emission to dominate over weak sources at that offset; (2) the data were taken during a series of Chandra non-detections; (3) the exposure was relatively short. 


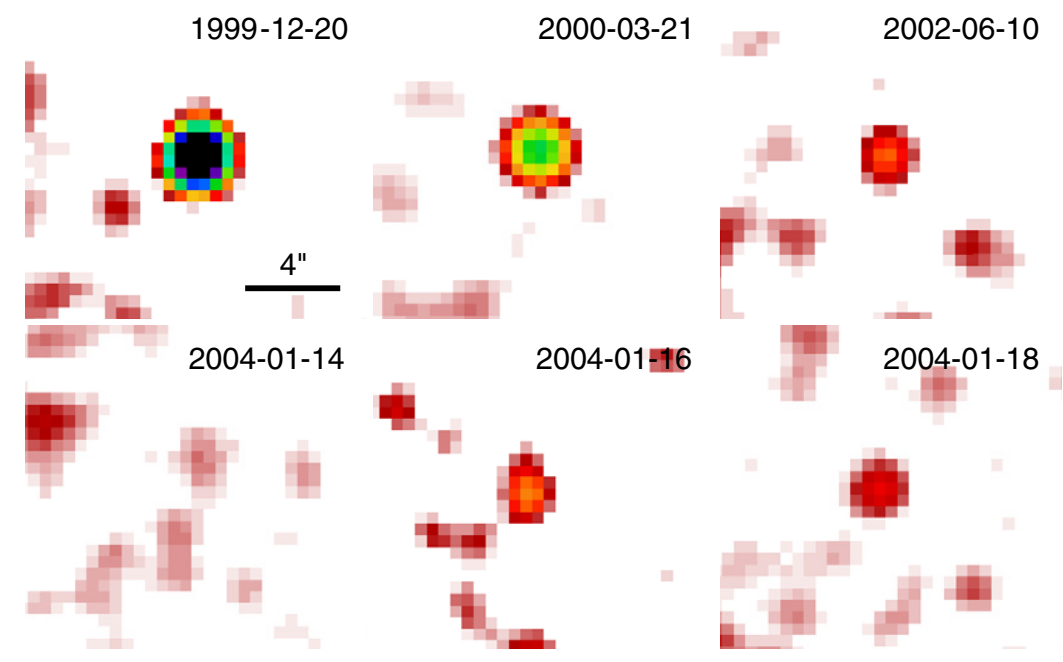

Figure 1. Field of view of the Chandra observations centered at the transient location. The $0.3-8 \mathrm{keV}$ images was smoothed with a 3 pixel Gaussian function in $d s 9$. (A color version of this figure is available in the online journal.)

\subsection{The Transient}

\subsubsection{EUVE}

EUVE pointed in the direction of A1795 seven times over $2.5 \mathrm{yr}$. For all the observations we analyzed the events recorded with the Deep Survey (DS) telescope (Bowyer \& Malina 1991) with the Lexan/Boron filter in the 0.0404-0.2816 keV (67-178 A) energy range. Significant emission was detected only in a $\sim 70.8 \mathrm{ks}$ observation on 1998 March 27 . In an earlier, very long observation ( $90 \mathrm{ks}$ ) on 1997 February 3 and in five shorter following pointings (up to $\sim 25 \mathrm{ks}$ ) in 1999, we were able to detect only emission coinciding with the center of the galaxy cluster, and in particular with the galaxy B2 1346+26 (the EUVE bright source $\mathrm{J} 1348+26.5 \mathrm{~B}$ ).

The cluster was the target of all the observations and, consequently, it was always on-axis, allowing us to consider the EUVE PSF as undistorted (the DS focal plane is curved, while the detector is flat). Since the distance of CXO J1348 from the central galaxy is $\sim 0$.7 and the angular resolution of $E U V E$ is $\sim 0.3$, the emission from the two objects overlaps.

To estimate the net flux associated with the transient we adopted the following procedure: We extracted the surface brightness profile (counts $\mathrm{s}^{-1} \operatorname{arcmin}^{-2}$ ) for both of the long observations (in 1997 and in 1998) using the reprocessed images downloaded from the HEASARC archive and a series of annular regions centered at the cluster position. The annuli were 0.4 wide. We used the deadtime-corrected exposure time from the header of the FITS files. A comparison of the two profiles shows that the transient is significant within the first 1'.5 from the cluster center. The counts associated with the cluster extend up to $4^{\prime}$. We extracted the net count rate from the inner 1'.5 region for both epochs and an annular region $\left(4^{\prime}-5^{\prime}\right.$ as inner/outer radii) as background. To generate a response file, we downloaded the effective area for the DS Lexar/Boron filter from the EUVE handbook.

Using Xspec for the observation in 1997, we created a spectrum with one bin covering the $0.0404-0.2816 \mathrm{keV}$ range. Using the APEC thermal model (with $k T=0.13 \mathrm{keV}$ and abundances $0.31 Z_{\odot}$; Bonamente et al. 2001) to model the soft cluster emission in the EUVE range, we found an observed flux of $(1.36 \pm 0.06) \times 10^{-12} \mathrm{erg} \mathrm{cm}^{-2} \mathrm{~s}^{-1}$. We generated a spectrum also for the 1998 observation using a combined model: the thermal model above and a BB component with a temperature of $k T=0.09 \mathrm{keV}$ (see Section 2.1.2). Subtracting the cluster contribution from the total flux, $(5.43 \pm 0.12) \times$ $10^{-12} \mathrm{erg} \mathrm{cm}^{-2} \mathrm{~s}^{-1}$, the observed flux from CXO J1348 is $(4.07 \pm 0.13) \times 10^{-12} \mathrm{erg} \mathrm{cm}^{-2} \mathrm{~s}^{-1}$. Using WebPIMMS, the flux was extrapolated to the Chandra $0.5-7 \mathrm{keV}$ energy range. As a result we found that the absorbed and unabsorbed fluxes are $(3.15 \pm 0.10)$ and $(3.41 \pm 0.10) \times 10^{-12} \mathrm{erg} \mathrm{cm}^{-2} \mathrm{~s}^{-1}$, respectively, at this time.

\subsubsection{Chandra}

A1795 is a familiar target for Chandra (Weisskopf et al. 2000): except for the first two pointings (PI: Fabian), the cluster has been used as a calibration source. Up to 2012 March, the telescope observed this field 43 times. Visual inspection of the images reveals that for only 26 pointings the position of $\mathrm{CXO}$ J1348 was within the boundaries of the ACIS (I or S) detectors. The list of useable observations is given in Table 1. As mentioned before, only exposures up to 2004 January 18 detect emission from CXO J1348 (see Figure 1 for a snapshot of all the Chandra observations from 1999 to 2004). Running wavdetect we found the following coordinates: $\alpha=13^{\mathrm{h}} 48^{\mathrm{m}} 49^{\mathrm{s}} .87$; $\delta=+26^{\circ} 35^{\prime} 57^{\prime \prime}$. 6 , with a systematic error of $00^{\prime} 6$. As explained in the CIAO 4.4 science thread, after correcting the aspect files we merged the ACIS-I data obtained in 2005 March and all the remaining ACIS-I and ACIS-S observations up to 2012. No significant excess was found at the transient position.

Before performing the data analysis of those observations with a detection, we re-generated the event 2 files using the chandra_repro script. We checked for the presence of flaring activity in the ACIS background: only very short time intervals were excluded from the following analysis. We selected the larger energy range $0.3-8 \mathrm{keV}$ to estimate the net count rate and the source significance, while we limited the spectral analysis to the artifact-free $0.5-7 \mathrm{keV}$ range. The spectral files for the source and the background as well as the response files were generated by the tool specextract, using a circular source extraction region with radius of $2^{\prime \prime}$ for the 1999 observation, when the source was brightest, $1^{\prime \prime} .5$ for the observation in 2000 , and $1^{\prime \prime}$. 0 for the remaining observations in 2002 and 2004. Smaller extraction regions were chosen in later epochs to reduce the contamination from the cluster X-ray light. A smoothed image shows that the 

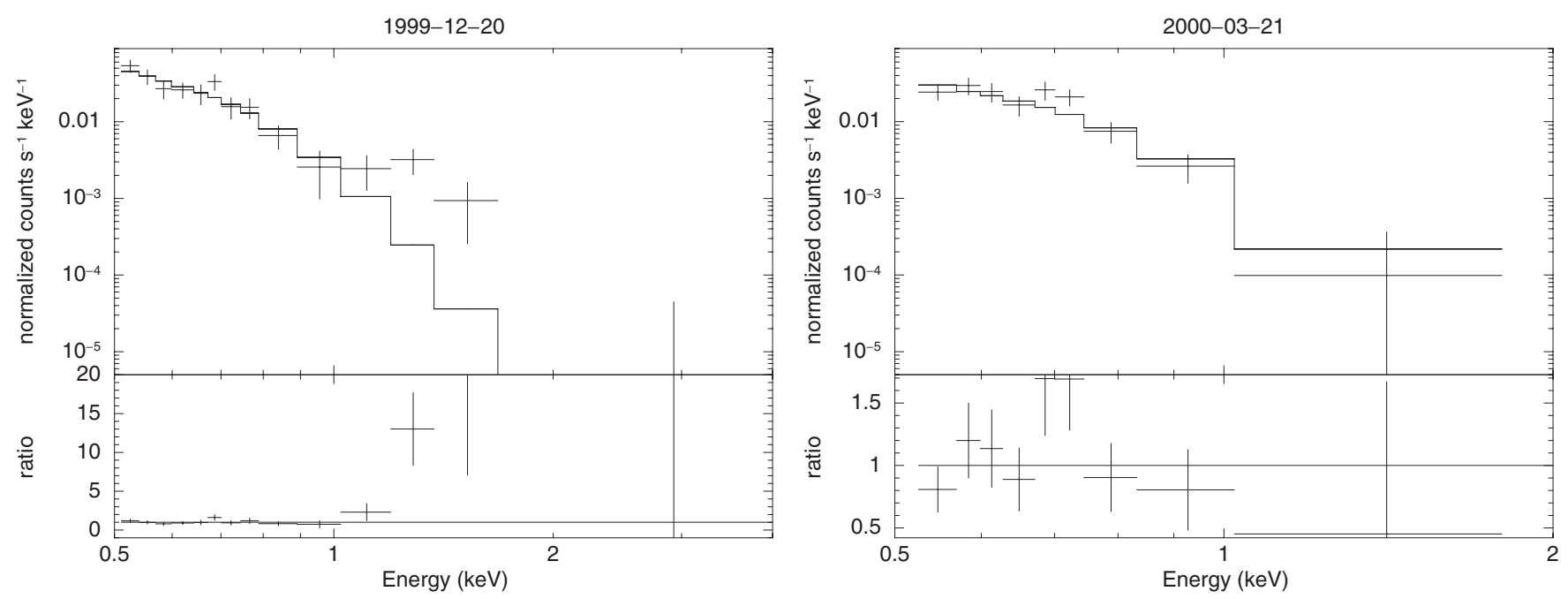

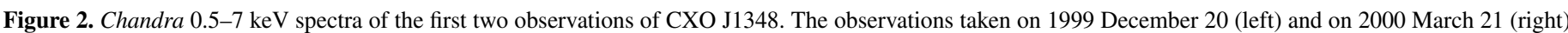
were fit using a blackbody model with $k T=0.10$ and $0.09 \mathrm{keV}$, respectively.

cluster emission still has a high gradient at the position of $\mathrm{CXO}$ J1348. For this reason, we selected as background regions 2 boxes along the isophotes of the smoothed emission, positioned on the two sides of the transient. The boxes are 4 " wide and $10^{\prime \prime}$ long. The source spectrum was grouped to a minimum number of 15 counts per channel when the source was bright (in 1999 and 2000).

The $0.5-7 \mathrm{keV}$ spectrum of CXO J1348 in 1999 can be adequately fitted $\left(\chi^{2}=15.5\right.$ for 12 dof) by a single $\mathrm{BB}$ model with $k T=0.10 \pm 0.01 \mathrm{keV}$ and absorption fixed at the Galactic level (left panel of Figure 2). The unabsorbed flux is $(2.8 \pm 0.4) \times 10^{-14} \mathrm{erg} \mathrm{cm}^{-2} \mathrm{~s}^{-1}$. There is a hint of an excess above $1 \mathrm{keV}$ and we added a second BB component to improve the fit. We found that the new fit $\left(\chi^{2}=10.3\right.$ for 10 dof) is obtained with $k T_{1}=0.08 \pm 0.02 \mathrm{keV}$ and $k T_{2}=$ $0.25 \pm 0.19 \mathrm{keV}$. The second component is not statistically significant as the probability obtained with the $F$-test is only $13 \%$. Using more complicated spectral models (diskbb or diskpbb) does not improve the fit, since they still do not compensate for the excess above $1 \mathrm{keV}$. Alternatively, a good fit can be obtained using a bremsstrahlung model. We found a temperature of $0.19 \pm 0.03 \mathrm{keV}\left(\chi^{2}=13.6\right.$ for $\left.12 \mathrm{dof}\right)$ and an absorbed flux of $(2.6 \pm 0.4) \times 10^{-14} \mathrm{erg} \mathrm{cm}^{-2} \mathrm{~s}^{-1}$. A fit with a power-law with a photon index of $\Gamma=4.35 \pm 0.27$ is also a reasonable representation of the X-ray spectrum $\left(\chi^{2}=11.6\right.$ for 12 dof $)$. Such a steep spectrum typically mimics thermal emission over the limited Chandra bandpass. Similar results have been found using an un-grouped spectrum and the $C$-statistic.

The observation obtained in 2000 was well fit $\left(\chi^{2}=7.8\right.$ for 7 dof) by an absorbed BB model (right panel of Figure 2). The temperature is $k T=0.09 \pm 0.01 \mathrm{keV}$ and the unabsorbed flux is $(2.1 \pm 0.4) \times 10^{-14} \mathrm{erg} \mathrm{cm}^{-2} \mathrm{~s}^{-1}$. No emission associated with the transient is observed above $2 \mathrm{keV}$. The use of a bremsstrahlung model does not fit the spectrum better: although the temperature $(k T=0.16 \pm 0.03 \mathrm{keV})$ is very similar to the 1999 observation, the residuals are higher $\left(\chi^{2}=9.8\right.$ for 7 dof $)$.

In 2002 the source faded significantly and only 27 net counts in the $0.3-8 \mathrm{keV}$ range are possibly associated with $\mathrm{CXO}$ J1348. The detection significance is $4.1 \sigma$ (that increases to $5.3 \sigma$ in the $0.3-2 \mathrm{keV}$ range). A spectral analysis using the $C$-statistic still shows the presence of the thermal BB component $(k T=0.07 \pm 0.03 \mathrm{keV})$ but at lower intensity $\left(2_{-1}^{+2} \times\right.$ $10^{-15} \mathrm{erg} \mathrm{cm}^{-2} \mathrm{~s}^{-1}$ in the $0.5-7 \mathrm{keV}$ range).

In all these trials we allowed an additional absorption at the cluster distance to vary, but its value was either small/unconstrained or did not improve the fit.

In 2004, the source was observed three times in a four day time span and it showed some signs of flaring activity: on January 14, the source was not significantly detected on the ACIS-S detector $\left(0.9 \sigma\right.$, with an upper limit on the count rate of $<4.7 \times 10^{-4}$ in the $0.3-8 \mathrm{keV}$ range), but it re-appeared on January 16 with a detection significance of $3.5 \sigma$ (ACIS-S net count rate $(1.4 \pm 0.4) \times 10^{-3}$ counts $\left.\mathrm{s}^{-1}\right)$. The transient was visible also on January 18 with a significance of $3.5 \sigma$ (ACIS-I net count rate $(1.3 \pm 0.3) \times 10^{-3}$ counts $\left.\mathrm{s}^{-1}\right)$. Due to the extremely poor statistics, no spectral analysis was performed. The unabsorbed flux in the $0.5-7 \mathrm{keV}$ range are $(4 \pm 1) \times 10^{-15} \mathrm{erg} \mathrm{cm}^{-2} \mathrm{~s}^{-1}$ and $(1.5 \pm 0.5) \times 10^{-14} \mathrm{erg} \mathrm{cm}^{-2} \mathrm{~s}^{-1}$, using the Chandra PIMMS tool $^{10}$ and assuming a BB model with a temperature of $0.09 \mathrm{keV}$ (similar to the values found in the 1999-2002 period). For the observation on January 14, we estimated a $3 \sigma$ upper limit of $<1.5 \times 10^{-15} \mathrm{erg} \mathrm{cm}^{-2} \mathrm{~s}^{-1}$.

Visual inspection of all the pointings starting in 2005 does not reveal the presence of the transient. We estimated an upper limit from the first pointing obtained on 2005 March 20 because the most sensitive detector ACIS-S was used. Since the cluster is the dominant source of emission, the upper limit remains high at $<1.4 \times 10^{-15} \mathrm{erg} \mathrm{cm}^{-2} \mathrm{~s}^{-1}$.

\subsubsection{VLA}

The field of A1795 has been extensively observed with the NRAO $^{11}$ VLA due to interest in its central bright radio galaxy, 1356+268 (e.g., Ge \& Owen 1993). Considering the time span after the detection of the transient X-ray source, we selected and analyzed archival VLA data of the field consisting of observations obtained at three epochs from 2000 October to 2005 October in the $\sim 5-8 \mathrm{GHz}$ range. All on-source exposures

\footnotetext{
10 Although the net count rates in 2002 and 2004 are almost identical, due to the evolution over time of the Chandra response matrices, PIMMS predicts higher fluxes for the ACIS-I detector.

11 The National Radio Astronomy Observatory is a facility of the National Science Foundation operated under cooperative agreement by Associated Universities, Inc.
} 
Table 2

Observation Log and Point Source $3 \sigma$ Limits from the VLA

\begin{tabular}{lcccrc}
\hline \hline $\begin{array}{l}\text { Date } \\
(1)\end{array}$ & $\begin{array}{c}\text { Program } \\
(2)\end{array}$ & $\begin{array}{c}\text { Freq. } \\
(3)\end{array}$ & $\begin{array}{c}\text { Beam } \\
(4)\end{array}$ & $\begin{array}{c}\text { Exp. } \\
(5)\end{array}$ & $\begin{array}{c}\text { Flux Density } \\
(6)\end{array}$ \\
\hline 2000 Oct 05 & AP405 & 8.46 & $11.50,9.05,-66.3$ & 590 & $<0.10$ \\
2003 Jul 11 & AF403 & 4.71 & $0.391,0.446,-33.5$ & 320 & $<0.32$ \\
2005 Oct 24 & AL663 & 8.46 & $15.70,5.55,-72.1$ & 63 & $<0.13$ \\
2005 Oct 24 & AL663 & 4.86 & $9.62,3.05,-72.5$ & 83 & $<0.18$ \\
\hline
\end{tabular}

Notes. Column explanations: $1=$ observation date; $2=$ program; $3=$ frequency in $\mathrm{GHz} ; 4=$ Gaussian restoring beam dimensions are the major axis ("), minor axis $\left({ }^{\prime \prime}\right)$, and the position angle in degrees; $5=$ exposure time in seconds; $6=$ detection limit $(3 \sigma)$ in $\mathrm{mJy}$.

were single snapshots lasting 1-10 minutes, using various VLA configurations. The data were calibrated in AIPS using standard procedures and self-calibration and imaging were performed with DIFMAP (Shepherd et al. 1994). No significant radio emission was detected at the position of CXO J1348 in any of the VLA observations with point source limits ranging from $<0.10$ to $<0.32 \mathrm{mJy}$ (see Table 2 ).

\subsection{The Host Galaxy}

A1795 has been observed extensively over the years in the optical by ground- and space-based telescopes. Coincident with the X-ray transient, we find a faint, resolved source which we shall assume to be the host galaxy of CXO J1348 (see Figure 3, left panel). Here we describe both new and archival observations of this galaxy, with the aim of constraining its distance and other basic properties (BH mass, nuclear activity, etc.).

The photometry of ground-based telescopes was performed using the DAOPHOT APPHOT photometry package in IRAF. Calibration was performed using field stars with reported fluxes in both the Two Micron All Sky Survey (Skrutskie et al. 2006) and the SDSS Data Release 9 Catalog (Ahn et al. 2012). The optical and infrared photometry of new and archival data is summarized in Table 3. The values have all been corrected for Galactic foreground extinction, assuming $E(B-V)=0.012$ (Schlafly \& Finkbeiner 2011) and a Milky Way extinction law with $R_{\mathrm{V}}=3.1$ (Cardelli et al. 1989).

\subsubsection{New Observations}

Observatorio Astronomíco Nacional/San Pedro Mártir (OAN/SPM) Johnson Telescope. Data were obtained with the multi-channel Reionization And Transients InfraRed camera (RATIR; Butler et al. 2012; Watson et al. 2012) mounted on the $1.5 \mathrm{~m}$ OAN/SPM telescope in Baja California, México. On 2013 February 12 and 19, we took a series of $60 \mathrm{~s}$ exposures with dithering between them in various filters (the number of exposures are indicate in parenthesis): $g$ (80), $r$ (80), $i$ (160), $Z$ (120), $J(120)$, and $H(22)$. Given the small galaxy size, a sky frame was created from a median stack of all the images in each filter. Flat-field frames consist of evening sky exposures. Due to lack of a cold shutter in RATIR's design, IR darks are not available. Laboratory testing, however, confirms that dark current is negligible in both IR detectors (Fox et al. 2012). The photometric images were reduced and co-added using standard CCD and IR processing techniques in IDL and Python.

Nordic Optical Telescope (NOT). We obtained three images in the $B$ and $i$ filters on 2012 March 20 with the ALFOSC camera
Table 3

Observation Log and Photometry in the UV/Optical/near-IR Bands

\begin{tabular}{|c|c|c|c|c|c|c|}
\hline $\begin{array}{l}\text { Telescope } \\
\text { (1) }\end{array}$ & $\begin{array}{l}\text { Date } \\
\text { (2) }\end{array}$ & $\begin{array}{l}\text { Instrument } \\
\text { (3) }\end{array}$ & $\begin{array}{c}\text { Filter } \\
\text { (4) }\end{array}$ & $\begin{array}{l}\text { Exp. } \\
\text { (5) }\end{array}$ & $\begin{array}{l}\text { Magnitude } \\
\text { (6) }\end{array}$ & $\begin{array}{l}\text { System } \\
\text { (7) }\end{array}$ \\
\hline HST & 1999 Apr 11 & WFPC2 & F555W & 300 & $23.02 \pm 0.13$ & V \\
\hline \multirow[t]{3}{*}{ VLT } & 2002 Jun $29^{a}$ & FORS1 & $U$ & 2840 & $23.43 \pm 0.28$ & V \\
\hline & & & $B$ & 1480 & $23.61 \pm 0.10$ & $\mathrm{~V}$ \\
\hline & & & $R$ & 800 & $22.14 \pm 0.05$ & $\mathrm{~V}$ \\
\hline \multirow[t]{4}{*}{ CFHT } & 2008 Aug 5 & MEGAPRIME & $g^{\prime}$ & 240 & $23.11 \pm 0.05$ & $\mathrm{AB}$ \\
\hline & & & $r^{\prime}$ & 120 & $22.22 \pm 0.05$ & $\mathrm{AB}$ \\
\hline & 2009 Jul 25 & MEGAPRIME & $g^{\prime}$ & 240 & $23.00 \pm 0.05$ & $\mathrm{AB}$ \\
\hline & & & $r^{\prime}$ & 120 & $22.15 \pm 0.05$ & $\mathrm{AB}$ \\
\hline \multirow[t]{3}{*}{ INT } & 2010 May 10 & WFC & $U$ & 400 & $23.59 \pm 0.16$ & V \\
\hline & & & $B$ & 400 & $23.42 \pm 0.09$ & $\mathrm{~V}$ \\
\hline & & & V & 400 & $22.37 \pm 0.06$ & V \\
\hline \multirow[t]{3}{*}{ NOT } & 2012 Mar 20 & ALFOSC & $B$ & 1500 & $23.40 \pm 0.15$ & V \\
\hline & & & $i^{\prime}$ & 900 & $21.40 \pm 0.15$ & $\mathrm{AB}$ \\
\hline & 2013 Mar 14 & MOSCA & $U$ & 6000 & $24.60 \pm 0.30$ & $\mathrm{AB}$ \\
\hline \multirow[t]{6}{*}{ Johnson } & 2013 Feb 12 & RATIR & $H$ & 1320 & $19.68 \pm 0.35$ & V \\
\hline & 2013 Feb 19 & & $g$ & 4800 & $23.14 \pm 0.20$ & $\mathrm{AB}$ \\
\hline & & & $r$ & 4800 & $22.16 \pm 0.08$ & $\mathrm{AB}$ \\
\hline & & & $i$ & 9600 & $21.91 \pm 0.06$ & $\mathrm{AB}$ \\
\hline & & & $Z$ & 7200 & $21.58 \pm 0.12$ & $\mathrm{AB}$ \\
\hline & & & $J$ & 7200 & $20.28 \pm 0.12$ & V \\
\hline
\end{tabular}

Notes. Column explanations: $1=$ telescope; $2=$ observation date; $3=$ instrument or camera; $4=$ filter; $5=$ exposure time in seconds; $6=$ magnitude; $7=$ photometric system (Vega or AB). The observations are ordered by date and frequency, starting with the bluer filter.

${ }^{\text {a }}$ This a sum of observations with equal exposures taken on 2002 June 8 and July 19.

and five images in the $U$ filter on 2013 March 14 with the MOSCA camera mounted on NOT (Karttunen 1993). Exposure times were 500, 300, and $600 \mathrm{~s}$, respectively. On both occasions, the sky conditions were photometric, however the seeing was variable. The frames were reduced and co-added using standard IRAF procedures (de-biasing, flatfield correction).

Large Binocular Telescope (LBT). On 2013 April 2 we obtained an optical spectrum with the Multi-Object Double Spectrograph (MODS1) instrument at the focus of the two $8 \mathrm{~m}$ mirrors of the LBT (Hill et al. 2000) using a 1".0 wide slit and the G400L and G670L grisms for the blue and red channels, respectively, covering the 3200-5800 $\AA$ and 5800-10000 range. The Clear filter was used for both grisms. The spectral resolution is $\delta \lambda^{*} c / \lambda \sim 200-400 \mathrm{~km} \mathrm{~s}^{-1}$, depending on the spectral region. Since MODS1 does not have an atmospheric dispersion corrector, the slit was oriented along the mean parallactic angle $\left(\right.$ P.A. $\left.=70^{\circ}\right)$. Conditions were clear with an average seeing always better than 1".5 FWHM and the observations were done in a sequence of four $1800 \mathrm{~s}$ exposures for a total integration time of $2 \mathrm{hr}$. The data were reduced by the LBT data center. Since the optical host is a dim object, we were able to detect only a weak continuum emission, with signal-to-noise ratio $(\mathrm{S} / \mathrm{N}) \approx 3$ per pixel $(\approx 8$ per resolution element) in the range from $6000-8000 \AA$. Due to the red galaxy color, the $\mathrm{S} / \mathrm{N}$ decreases as a function of wavelength and no significant signal is detected below $\approx 4000 \AA$. No significant features are observed over the range from 4000-9500 $\AA$, neither in absorption nor emission. Specifically, for the region from $\approx 6000-8000 \AA$, we limit the flux from any emission line to be $f \lesssim 10^{-17} \mathrm{erg} \mathrm{cm}^{-2} \mathrm{~s}^{-1}$ (assuming a line width of several hundred $\mathrm{km} \mathrm{s}^{-1}$, corresponding to our instrumental resolution). 

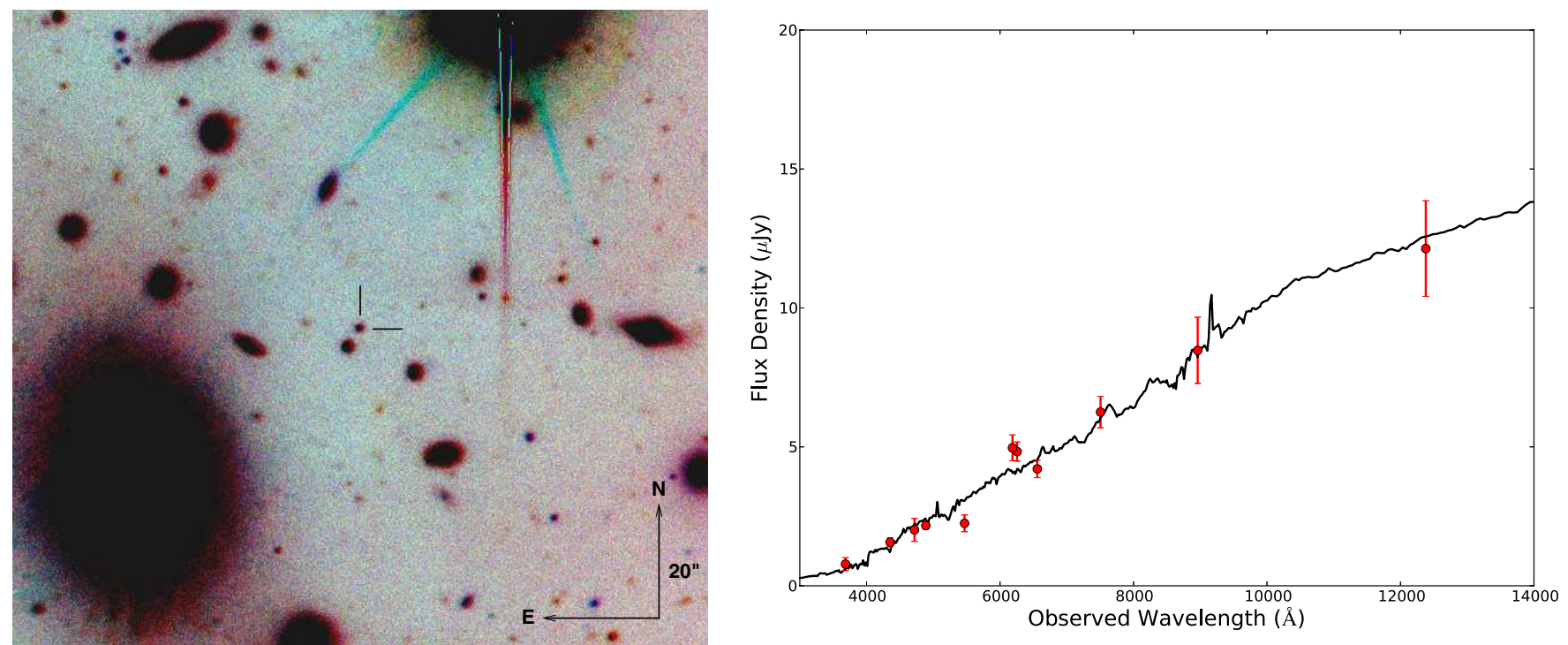

Figure 3. Left: false color image combining CHFT $g^{\prime}$ and $r^{\prime}$ and NOT $i^{\prime}$ bands. The host galaxy of CXO J1348 is marked at the center of the image. Right: SED obtained by combining all the new and archival optical and near-IR photometric measurements. The black line indicates the best fit found by running the photo-redshift code EaZy, using an evolved stellar population model at redshift $z=0.13_{-0.05}^{+0.18}$.

(A color version of this figure is available in the online journal.)

\subsubsection{Archival Images}

Hubble Space Telescope (HST). Although A1795 has been the target of many HST pointings, due to the very small field of view CXO J1348 fell on a detector only in a single set of observations. On 1999 April 11 the telescope observed the source position using the Wide Field Planetary Camera 2 (Holtzman et al. 1995 ) for $300 \mathrm{~s}$, both with the F555W and the F814W filters. Unfortunately, at that time the drizzling technique to remove cosmic rays was not adopted and single images (per filter) were taken. An optical counterpart at the position of the X-ray transient is detected on the WF2 chip in both filters. A visual inspection of the processed images revealed the presence of cosmic rays very close to the counterpart in the F814W image. The photometry in this filter is, thus, unreliable. The estimated flux in the F555W filter is corrected for the finite aperture using Table 2 in Holtzman et al. (1995).

We also check the morphology of the optical source by comparing it with an artificial PSF. We used the web interface of the PSF modeling tool Tiny Tim (Krist et al. 2011) to generate a PSF located at the same position on the WF2 chip of the F555W filter, and we assumed a spectrum described by a powerlaw with index -1 (changing the spectral slope does not alter the shape of the PSF significantly). We selected the F555W filter because there were no identifiable cosmic rays close to the source. Since the object is very dim and the PSF is undersampled, we smoothed the image using a Gaussian function with a 2 pixel kernel radius. We extracted a brightness profile along the east-west direction (the diffraction spikes do not contribute since they are tilted by $10^{\circ}$ ). The profile of the source was compared with that of the generated PSF, and smoothed with the same kernel function. The Gaussian function that describes the artificial PSF has a FWHM of 0'.28, while the source brightness profile has a larger width $(\mathrm{FWHM}=0.40)$. This indicates that the source is spatially resolved, suggesting a galaxy-like morphology. A comparison between the images in the two filters shows that there is some additional emission above the PSF and $3 \sigma$ above the local background located at P.A. $=215^{\circ}$ and with extension of 0 '. 6 . The source measures 1 .'05 along this angle and 0.7 perpendicularly. The short exposure of the two images did not allow us to speculate on the nature of this feature.

Very Large Telescope (VLT) and Canada-France-Hawaii Telescope (CFHT). The optical counterpart at the position of CXO J1348 has been observed with the FORS1 camera on the VLT (Nicklas et al. 1997) on 2002 June 8 and July 19, and the MegaPrime/MegaCam on CFHT (Boulade et al. 2003) on 2008 August 5 and 2009 June 25. Frames from the two telescopes have been corrected by means of bias or dark frames and response was normalized by means of flat-field frames. Given the lack of variability due to the temporal proximity of the two sets of VLT data, we decided to sum the observations to increase the signalto-noise ratio, in particular in the $U$ filter where the source was barely detected.

Isaac Newton Telescope (INT). Querying the online catalogs at $H E A S A R C$ we found that photometric measurements for an object consistent with the Chandra source were already available. The optical source was observed as part of the WIdefield Nearby Galaxy-cluster Survey (WINGS; Varela et al. 2009) between 2000 and 2001. The automatic software that run the analysis in the $B$ and $V$ filters determined that the object (WINGS $\mathrm{J} 134849.88+263557.5$ ) can be classified as a galaxy. The galaxy cluster has been extensively observed through the years, from 1992 to 2010, using the Wide Field Camera on the INT (Lewis et al. 2000). While the images in the $r$ and $i$ filters suffer from bad fringing, the observations in the $U, B$, and $V$ can be used to extract valuable photometry. The source does not show any sign of variability. In Table 3 we report the values obtained in the 2010 May campaign, a period not covered by other groundbased telescopes.

Spitzer. Only one imaging data set covering the location of CXO J1348 is available in the Spitzer archive. It was taken on 2010 August 8 with the IRAC instrument (Fazio et al. 2004) in both the $3.6 \mu \mathrm{m}$ and $4.5 \mu \mathrm{m}$ bandpasses. A visual inspection reveals that only in the $3.6 \mu \mathrm{m}$ (a $380 \mathrm{~s}$ exposure) mosaic image there is a source at the X-ray transient position detected at the $2 \sigma$ level, while in the $4.5 \mu \mathrm{m}$ bandpass the 
significance is $1 \sigma$. Unfortunately, the relatively short exposures and the contamination from the galaxy at the center of the cluster do not allow us to extract a reliable and meaningful photometric measurement: using circular regions for both the source and the background with a 2 pixel radius and applying the aperture corrections listed in Section 4.10 of the IRAC instrument handbook, we obtained flux densities of $12.8 \pm$ $7.7 \mu \mathrm{Jy}$ and $3.4 \pm 3.0 \mu \mathrm{Jy}$ in the $3.6 \mu \mathrm{m}$ and $4.5 \mu \mathrm{m}$ bandpasses, respectively. Due to the large errors, we do not use these values in the analysis.

Swift Ultra-Violet/Optical Telescope (UVOT). A1795 was observed with UVOT (Roming et al. 2005) in the UVW1 filter on board the Swift satellite on 2005 November 12. We used uvotimsum to combine the total of $\sim 24 \mathrm{ks}$ exposure obtained over three orbits. Running uvotsource we found that no significant emission above the background was observed at the $\mathrm{X}$-ray position with an upper limit, $m_{\mathrm{UVw} 1}<22.4$ (in the Vega system).

\subsubsection{Characteristics of the Host Galaxy}

Since no spectroscopic redshift of the host galaxy is available, we combined photometry from archival VLT, CFHT, INT, and HST data with new observations from RATIR and NOT to produce a detailed SED of the host galaxy (right panel of Figure 3).

We fitted the SED using the EaZy code (Brammer et al. 2008) and models from Bruzual \& Charlot (2003): We considered different kinds of galaxy spectral templates (elliptical, early and late spirals, irregular and starburst galaxies), set at different ages and with various metallicities and star formation rates. Although many templates provide a reasonable fit of the putative host galaxy SED, the best fit is obtained by either an elliptical or S0 template, both dominated by an old, evolved stellar population, located at a redshift of $z=0.13_{-0.05}^{+0.18}(68 \%$ confidence level). The derived photometric redshift is compatible with the average value of the cluster redshift $(z=0.062476)$ and the dispersion of the velocities of the cluster brightest galaxies, whose redshifts range from 0.054 and 0.068 (Smith et al. 2004).

The inferred de-reddened magnitude at the cluster redshift is $M_{\mathrm{B}} \sim-13.8\left(M_{\mathrm{R}} \sim-15.1\right)$ and the estimated scale is $1.188 \mathrm{kpc} \operatorname{arcsec}^{-1}$ (Wright 2006). Based on the result of the spatial analysis of the $H S T$ data, this corresponds to a minimum radial extension of $0.4 \mathrm{kpc}$ (and up to $0.7 \mathrm{kpc}$ along the putative feature observed to the south-west of the source). Thus, both the source brightness and its radial extent suggest that we are observing either a compact elliptical galaxy or a spiral galaxy with a small bulge and even dimmer spiral arms. Both scenarios are supported by the reasonable fit obtained using an elliptical or S0 template for the estimate of the photo-redshift.

The uncertainties on the photometric redshift are somewhat large in the upper end side: the $68 \%$ error puts the host galaxy at $z=0.31$, an increase by a factor of 5.8 in the luminosity distance and of 3.8 in the angular scale. Thus, the source would be 3.8 magnitude brighter and $1.5 \mathrm{kpc}$ in size.

The assumption that the host galaxy belongs to the cluster A1795 is supported by its projected location, very close to the main galaxy of the cluster, and by the richness of the cluster. Querying the SDSS catalog for an area with a radius of $20^{\prime}$, where previous works (see, e.g., Oegerle \& Hill 2001) have found the majority of the cluster components, we find that more than 150 galaxies (corresponding to three out of four galaxies with a spectroscopic measurement) have a redshift compatible with the cluster. We cannot rule out entirely the possibility that the host galaxy belongs to a more distant group or is a background object. Of the remaining galaxies with an estimate of their distance, 20 sources have a redshift of $z=0.11$, indicating that another cluster may be located behind A1795, $\sim 15$ objects have redshift more evenly distributed in the ranges $0.15-0.20$ and $0.24-0.31$, while a few other sources are located at cosmological distances.

\section{ORIGIN OF THE UV/X-RAY TRANSIENT}

The spatial analysis of the HST and ground-based data shows that the optical counterpart at the position of the UV/X-ray transient is not a point source. This excludes any local origin (e.g., explosion in a classical or a recurrent nova system, X-ray burst on the surface of a neutron star, etc.) and leads to the conclusion that we are observing an extragalactic object, i.e., a distant host galaxy.

The probability of chance alignment of the optical source with a Chandra position is very low. Following Bloom et al. (2002) and Perley et al. (2012) an estimate of the probability of chance association $P$ can be expressed as

$$
P=1-\exp ^{-A \rho}
$$

Here, $A$ is the area on the sky encompassing the X-ray and the optical sources, while $\rho$ is the sky density of objects of equal or greater brightness. Very conservately we choose a circle with a radius of $2^{\prime \prime}$ as the area, that corresponds to the largest $\mathrm{X}$-ray flux extraction region. There are other factors that might contribute to the size of that circle, like the Chandra astrometry error (typically of the order of 0.'6) or the HST PSF of the object ( 0.4 , see above), but their contribution is not significant when compared to the size of the extraction region. We queried the SDSS catalog to have a list of sources brighter than the host galaxy $23.14 \mathrm{mag}$ in the $g^{\prime}$ filter in a circle with a radius of $15^{\prime}$ and centered at the transient position. Since we found $\sim 2000$ objects, the chance probability is, thus, $<0.01$. Assuming the quadratic sum of Chandra and HST astrometric errors only, the probability is $\sim 0.001$.

A similar argument can be done to estimate the chance probability that the transients observed by EUVE and Chandra are actually the same object. This assumption is hampered by the fact that there is a 21 month gap between the 1998 March $E U V E$ and the 1999 December Chandra observations, which includes multiple $E U V E$ non-detections. The difference with the previous approach is that there are no sources in both fields brighter than the transient. The only other point-like object detected by EUVE is EUVE J1348+26.5A, a Seyfert 1 galaxy located at 5'.8 from the transient (from the second $E U V E$ right angle program catalog in Christian et al. 1999). This object is also the second brightest point source in all the combined Chandra fields of A1795, after the transient. Assuming an extraction region of 1'.5 (see the EUVE analysis in Section 2.1.1), the estimated chance probability is $\sim 0.065$. This does not include the likelihood of another (unrelated) high-amplitude flare in the field, however. While difficult to quantify, we can use results from the GALEX Time Domain Survey (Gezari et al. 2013) and the XMM-Newton Slew Survey (Saxton et al. 2008) to estimate the probability of an unrelated transient source in our EUVE images. The sky density of highly variable ( $\triangle \mathrm{mag} \geqslant 2$ in the GALEX NUV filter) $\mathrm{M}$ dwarf flares (the dominant class of such dramatic variability) is $\sim 5 \mathrm{deg}^{2} \mathrm{yr}^{-1}$ (Gezari et al. 2013). Given the duration of the $E U V E$ exposure $(70.8 \mathrm{ks})$ and the astrometric uncertainty (1.5), the likelihood of chance detection of an unrelated source is only 
$\sim 2 \times 10^{-5}$. Similarly, Kanner et al. (2013) calculate the rate of X-ray transients of comparable brightness to CXO J1348 is $3 \times 10^{-3} \mathrm{deg}^{-2}$. The implied probability of detecting an unrelated source is again very small, $\sim 6 \times 10^{-6}$. We conclude the EUVE detection is highly likely associated with CXO J1438.

Here we consider four possible classes of extragalactic transients known to produce the observed degree of X-ray variability: a gamma-ray burst (GRB), a supernova, a sudden outburst from an active galactic nucleus (AGN), or a TDE.

\subsection{GRB/Supernova Scenario}

The most compelling lines of evidences against the GRB/supernova scenario are: (1) the length of the UV/X-ray light curve; (2) the short term variability at late time; (3) the shape of the X-ray spectrum. Below we discuss each in more detail.

CXO J1348 is detected for approximately six years, a period over which the intensity decays by three orders of magnitude (from the EUVE detection in 1998 and the Chandra upper limit in early 2004 there is a factor of 2300). Swift monitoring of hundreds of GRB afterglows (D'Avanzo et al. 2012) shows similar decrease in brightness, but they do not last as long: the longest light curves (e.g., GRB 060729) span only a time frame of a few months before reaching a flux level of $\sim 10^{-14} \mathrm{erg} \mathrm{cm}^{-2} \mathrm{~s}^{-1}$ (a value similar to the last detection of CXO J1348 by Chandra in 2004).

On the contrary, supernovae can be detected in the X-rays for decades (Dwarkadas \& Gruszko 2012) but the light curve does not decay as much and, in some cases, it rises over time due to the interaction of the shock waves with the circumstellar medium. As mentioned in Section 2, the Chandra data taken $5 \mathrm{yr}$ after the first $E U V E$ observation show dramatic variability over a time of four days. This kind of late time variation is strong evidence against the supernova scenario, since a typical X-ray light curve is not expected to show fast flaring activity many years after the original explosion.

The result of the Chandra spectral analysis can be used to rule out the GRB afterglow scenario. Their X-ray spectra are fitted by a power-law model with a flat spectral slope $(\Gamma \lesssim 2$, Butler 2007), while a small fraction (10\%) of the afterglows have a prominent residuals near $1 \mathrm{keV}$ that can be fitted with an additional BB model. On the contrary, the first observation with Chandra of CXO J1348 was fit with a much steeper power-law $(\Gamma \sim 4.3)$ or by simple thermal model, as are all the following spectra.

Furthermore, the host shows no evidence for star formation. Long GRBs are found exclusively in star-forming galaxies (e.g., Fruchter et al. 2006; Savaglio et al. 2009) and core-collapse supernovae are the only ones for which X-ray emission has been detected.

\subsection{AGN Scenario}

The lack of emission lines in the optical LBT spectrum might be intrinsic, as seen in blazars, a sub-class of radio-loud AGNs (Urry \& Padovani 1995), or because the lines were too dim to be detected. Optical broad or narrow emission lines, like $\mathrm{H} \alpha$, $\mathrm{H} \beta$, etc. are observed in a large variety of AGNs, all of them showing some X-ray variability. At the redshift of the cluster, our limits on any $\mathrm{H} \alpha$ emission correspond to a luminosity of $L_{\mathrm{H} \alpha}<8 \times 10^{37} \mathrm{erg} \mathrm{s}^{-1}$. This value is several orders of magnitude less than the $\mathrm{H} \alpha$ luminosities derived from Greene \& Ho (2007) for active galaxies with low-mass BHs.

\subsubsection{Radio-loud AGN/Blazars}

The radio emission observed in these objects is produced by accelerated particles that travel in collimated, relativistic jets and emit synchrotron radiation. As mentioned earlier, the object is not found in VLA archival images down to a $3 \sigma$ flux limit of $<0.1 \mathrm{mJy}$. At a distance of $z \sim 0.07$, the radio luminosity is $L_{8.5 \mathrm{GHz}}<10^{38} \mathrm{erg} \mathrm{s}^{-1}$. This makes the putative AGN a radio-dim (or even a radio-quiet) source. Indeed, observations of the Hubble deep fields at $8.5 \mathrm{GHz}$ show that all the radio sources have fluxes below the above upper limit and they are not identified with quasars but rather with starforming galaxies, bright field elliptical and late-type galaxies with evidence of nuclear activity (Seyferts) and field spiral galaxies (Richards et al. 1998). The upper limit excludes not only radio-loud galaxies but also radio-loud quasars (blazars): their radio luminosities start two orders of magnitude higher (Donato et al. 2001; Giommi et al. 2012) and the broadband radio-to-optical spectral index $\left(\alpha_{\text {ro }}\right)$ between $8.46 \mathrm{GHz}$ and $5500 \AA$ is higher than the upper limit of 0.28 found for CXO $\mathrm{J} 1348$, a value that places the putative AGN in the radio-quiet regime.

Furthermore, blazars typically occur in very massive, luminous galaxies (see, e.g., Urry et al. 2000) and there is some evidence that they might be considered quasi-standard candles, with optical absolute magnitude greater than -24 , many orders of magnitude brighter than what is observed in this host galaxy.

\subsubsection{Radio-quiet AGN/Seyfert}

Among the radio-quiet AGNs, like quasars, Seyfert galaxies, and low-ionization nuclear emission-line regions, narrow-line Seyfert 1 (NLSy1) galaxies are the most variable, although the changes are not as dramatic as in CXO J1348. In NLSy1 galaxies, the variability can be explained as due to changes in the properties of the absorber surrounding the inner BH such as column density, ionization parameter, and covering factor (e.g., Grupe et al. 2012). The highest variability was seen in WPVS 007 (Grupe et al. 1995) with a decrease in flux by a factor of 400 in the soft band in a $3 \mathrm{yr}$ time span, while fast variability has been observed in NGC 4051 (McHardy et al. 2004) with a change of the X-ray flux by a factor of 10 in a few days. This could explain changes seen in Chandra data alone, but not if we assume that the EUVE transient is the same source.

One difference between $\mathrm{CXO} \mathrm{J} 1348$ and the radio-quiet AGNs is the shape of the X-ray spectrum: radio-quiet AGNs have their spectra described by a power-law with spectral index in the range 1.5-3.5, irrespective if the source harbors a SMBH or an IMBH, and only the more luminous (and more massive) objects show the presence of the soft excess with temperature above $0.1 \mathrm{keV}$ (Brandt et al. 1997; Grupe et al. 1995; Porquet et al. 2004; Miniutti et al. 2009; Pian et al. 2010; Dong et al. 2012). The NLSy1 galaxies do show a steep spectrum in the soft band (some of the ROSAT spectra have slopes above 4), but they are detected at higher energies as well: the $A S C A$ spectra can be fitted in first approximation with a combination of a power-law model and a thermal BB component with temperature in the range $0.1-0.2 \mathrm{keV}$ (Leighly 1999). On the contrary, the spectrum of CXO J1348 does not show any emission above $3 \mathrm{keV}$ and, consequently, no additional power-law model is needed.

At optical wavelengths, Zhou et al. (2006) found that in a sample of $\sim 2000$ optically selected NLSy1 in the SDSS, their 


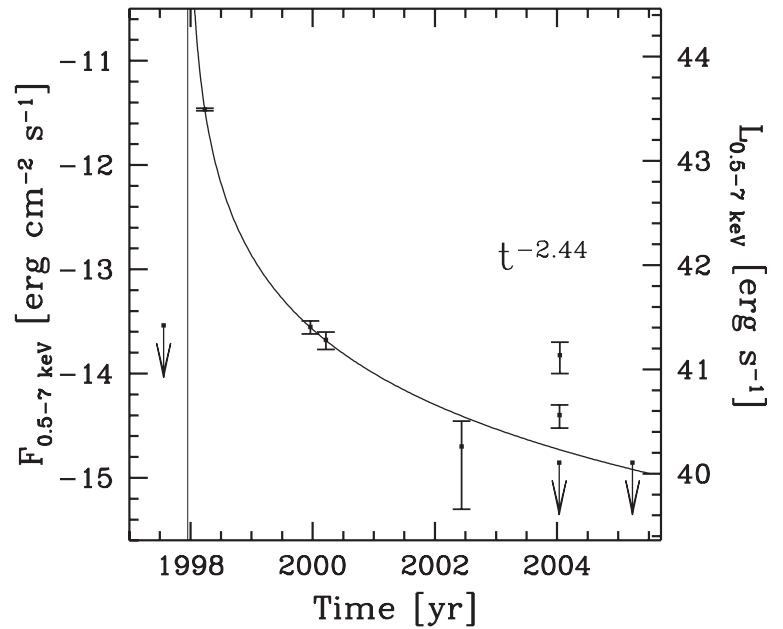

Figure 4. Light curve for the UV/X-ray flare. The first point is an upper limit from ROSAT, while the first detection is from EUVE, and converted to the Chandra $0.5-7 \mathrm{keV}$ energy range assuming a blackbody model with temperature of $0.09 \mathrm{keV}$, as seen in following observations by Chandra. The last point is an upper limit from the ACIS-S observation on 2005 March 20.

absolute magnitude in the $g^{\prime}$ filter is in the range $-18 \lesssim M_{\mathrm{g}^{\prime}} \lesssim$ -26 , while the host galaxy of CXO J1348 is much dimmer $\left(M_{\mathrm{g}^{\prime}} \sim-14.1\right)$.

\subsection{TDE Scenario}

The analysis of the combined Chandra and EUVE light curve shows that the brightness decay in the $0.5-7 \mathrm{keV}$ range is consistent with a power-law decline in time, characterized by $a \times\left[\left(t-t_{\mathrm{D}}\right) /\left(t_{0}-t_{\mathrm{D}}\right)\right]^{-n}$ law, where $a$ is the normalization and $t_{\mathrm{D}}$ and $t_{0}$ are the time of the disruption and of the flare's peak, respectively. Leaving all parameters free to vary, we performed a least-squares fit of the decay (reduced $\chi^{2}=3.1$ for 5 dof) and we found the following set of parameters: $a=$ $(1.51 \pm 0.75) \times 10^{-13}, n=2.44 \pm 0.40$, and $t_{\mathrm{D}}=1997.957 \pm$ 0.115 (corresponding to 1997 December 17 with an uncertainty of $\sim 42$ days). While the value of $t_{0}$ remains unconstrained, $t_{\mathrm{D}}$ is consistent with the upper limit on the ROSAT data taken on 1997 July 23. However, the shape of the decline, shown in Figure 4, does not depend significantly on $t_{0}$. This fit indicates that the disruption happened approximately three months before the EUVE detection, further strengthening the association between the UV and X-ray transient. The slope has a value that is fully compatible with observations of other candidate TDEs (Komossa \& Greiner 1999; Cappelluti et al. 2009; Gezari et al. 2009).

We also fitted the light curve using the Chandra data only to understand (1) if the assumptions we made to calculate the $E U V E$ point, obtained by extrapolating the UV analysis to the Chandra energy range, are correct; (2) if the EUVE point is on the same slope of the Chandra light curve; (3) if the theoretical models that predict a slope with index $n=5 / 3$ at later time (from days up to few months after the event) are correct. Those models predict also that the slope is flatter immediately after the explosion (Lodato et al. 2009; Gezari et al. 2009; Strubbe $\&$ Quataert 2009) with deviations from the $t^{-5 / 3}$ law more pronounced for more centrally concentrated stars (e.g., solar type).

Unfortunately, the large relative errors on the X-ray fluxes do not allow us to put firm constrains on $t_{\mathrm{D}}$ when fitting only the Chandra data. Leaving the parameters free to vary, we found that the slope becomes flatter $(n=1.52 \pm 0.50)$ but $t_{\mathrm{D}}=1999.11 \pm 1.22$, i.e., consistent with a pre- $E U V E$ disruption time within $1 \sigma$, although the errors are large. We forced the fit by imposing $t_{\mathrm{D}}$ to be between the ROSAT upper limit in 1997 July and the detection by EUVE in 1998 March. The slope becomes steeper again, ranging from $n=2.71 \pm 0.36$ when $t_{\mathrm{D}}$ is fixed immediately after the ROSAT observation to $n=2.26 \pm 0.29$ when $t_{\mathrm{D}}$ is set just a few days before the first detection. These values are fully compatible with the fit obtained using the $E U V E$ data. In these trials we also calculated the flux at the time of the EUVE observation and found that only by setting $t_{\mathrm{D}}$ in the last two months of 1997 will the predicted flux (a few $\times 10^{-12} \mathrm{erg} \mathrm{cm}^{-2} \mathrm{~s}^{-1}$ ) have been compatible with what we measured. These results reinforce the idea that (1) the disruption event happened a few months before the EUVE detection; (2) the light curve peaked before the first observation; (3) the slope of the light curve decay is slightly steeper than most simple theoretical predictions.

\section{DISCUSSION}

\subsection{Estimate of the Black Hole Mass}

Based on the photo-redshift, the derived absolute magnitudes indicate that we are observing a very dim host, regardless of the exact adopted redshift. If we make the simplest assumption that the host galaxy lies at the distance of A1795, we derive an absolute magnitude of $M_{\mathrm{g}^{\prime}} \sim-14.1\left(M_{\mathrm{B}} \sim-13.8\right.$, $\left.M_{\mathrm{R}} \sim-15.1\right)$.

Very recently Graham \& Scott (2013) have shown that the scaling relation between $\mathrm{BH}$ mass and host spheroid luminosity is bent when core-Sérsic and Sérsic galaxies are considered. The first class contains galaxies whose spheroidal component is thought to be created by simple additive dry merger events and is more typical for elliptical galaxies, while in the second class the spheroidal component is possibly created by gas-rich processes, something more typical of spiral galaxies. From the template used to estimate the photo-redshift we extrapolated the expected near-IR $K$-band flux $\left(\mathrm{F}_{\mathrm{K}}=14 \pm 5 \mu \mathrm{Jy}\right.$ corresponding to $\left.m_{\mathrm{K}}=19.2 \pm 0.4\right)$. Assuming the cluster distance, the absolute magnitude is $M_{\mathrm{K}} \sim-18.0 \pm 0.4$. Unfortunately, there are no sources included in Graham \& Scott (2013) with such a low luminosity. In their samples, the core-Sérsic galaxies have $M_{\mathrm{K}} \gtrsim-22$ and $M_{\mathrm{B}} \gtrsim-18$, while the Sérsic galaxies have $M_{\mathrm{K}} \gtrsim-20$ and $M_{\mathrm{B}} \gtrsim-16$. As the authors argue, it is not clear if the relation may hold for dimmer objects. Furthermore, recent studies (McGee 2013) show that in clusters the central and satellite galaxies may follow distinctly separate scaling relations. This can exacerbate the uncertainties in the relation on the faint end. Since the morphology of this host galaxy is undetermined, we tried both relations and found, as expected, larger $\mathrm{BH}$ mass estimates for the core-Sérsic cases $\left(\log \left(M_{\mathrm{BH}} / M_{\odot}\right)=6.0 \pm 0.9\right.$ and $\left.5.1 \pm 1.0\right)$, with respect to the Sérsic cases $\left(\log \left(M_{\mathrm{BH}} / M_{\odot}\right)=2.5 \pm 1.5\right.$ and $\left.2.5 \pm 1.0\right)$. The two estimates are obtained using the $K$ and $B$ magnitudes, respectively. The $\mathrm{BH}$ mass in the Sérsic cases are very low and this might indicate that the scaling relation is not valid for objects with very low absolute magnitude. As mentioned earlier, the SED of the host galaxy was best fit using a galaxy template with an old, evolved stellar population. This suggests that a core-Sérsic galaxy is more appropriate.

In another recent work, Kormendy \& Ho (2013) used more accurate $\mathrm{BH}$ masses, partly because of improvements in models that include dark matter. They considered only classical bulges, 
corresponding to the core-Sérsic galaxies defined in Graham \& Scott (2013), and ignored the pseudo-bulges, galaxies that fit the Sérsic definition. Following their proposed $M_{\mathrm{BH}}-M_{\mathrm{K} \text {,bulge }}$ relation we found that the mass of the $\mathrm{BH}$ is $\log \left(M_{\mathrm{BH}} / M_{\odot}\right)=$ $5.7 \pm 0.5$. We would like to stress that also in this work there are no galaxies below $M_{\mathrm{K}} \sim-19$ (corresponding to M32, the satellite of the Andromeda galaxy) and the relation is not tested at such low luminosities.

The estimated mass is higher if the upper bound in the photoredshift $(z=0.31)$ is considered. As mentioned above, the absolute magnitude would decrease by $3.8 \mathrm{mag}$, corresponding to $M_{\mathrm{K}} \sim-21.8$. For this luminosity, the relations for coreSercic galaxies in Graham \& Scott (2013) and classical bulges in Kormendy \& Ho (2013) would have given a value for the $\mathrm{BH}$ mass of $\log \left(M_{\mathrm{BH}} / M_{\odot}\right)=7.6 \pm 0.5$ and $7.5 \pm 0.5$, respectively.

Combining the results from Graham \& Scott (2013) and Kormendy \& Ho (2013) predictions for core-Sérsic galaxies (classical bulges) we argue that, based on the brightness of the host galaxy and the cluster A1795 distance, we are observing an intermediate mass $\mathrm{BH}$, with mass in the range $\sim 10^{5}-10^{6} \mathrm{M}_{\odot}$. Throughout the rest of the paper, we assume an average value of $10^{5.5} M_{\odot}$.

\subsection{Dynamics}

The analysis of the UV/X-ray light curve did not allow us to put constraints on the peak time $t_{0}$. Theoretical models of TDEs predict that after the encounter half of the stellar debris is unbound from the $\mathrm{BH}$ and leaves the system, while the other half returns to the pericenter after a minimum (or fallback) time that is equivalent to the gap between the moment of the disruption $t_{\mathrm{D}}$ and $t_{0}$. This gap depends on the geometry of the encounter, the nature of the disrupted star, and the $\mathrm{BH}$ mass. Following Ulmer (1999) and Maksym et al. (2010), we define $r_{*} \equiv R_{*} / R_{\odot}$ and $m_{*} \equiv M_{*} / M_{\odot}$, where $R_{*}$ and $M_{*}$ are the radius and mass of the disrupted star, the penetration factor $\beta=R_{\mathrm{t}} / R_{\mathrm{p}}$, where $R_{\mathrm{p}}$ and $R_{\mathrm{t}}$ are the periastron and tidal radius of a $\mathrm{BH}$, whose mass $M_{6}$ is in units of $10^{6} M_{\odot}$. Then,

$$
t_{\text {fallback }}=\left(t_{0}-t_{\mathrm{D}}\right)=0.11 k^{-3 / 2} M_{6}^{1 / 2} \beta^{-3} r_{*}^{3 / 2} m_{*}^{-1} \mathrm{yr} .
$$

While Li et al. (2002) proposed that the parameter $k$ ranges from 1 for a non-rotating star to a more favorable value of 3 for a star which is spun up near the point of disruption, more recent work (Lodato et al. 2009) suggests that the spin-up may not be a significant factor in the fallback evolution. As summarized by Maksym et al. (2010), the factor $r_{*}^{3 / 2} m_{*}^{-1}$ can be simplified as $m_{*}^{1 / 2}$ for main-sequence stars with $M_{*}<1 M_{\odot}$ and $m_{*}^{1 / 8}$ for main-sequence stars with $M_{*}>1 M_{\odot}$. Furthermore, recent analytic models (e.g., Stone et al. 2013) suggest a weaker dependence of fallback time on the penetration factor; however, this will not significantly affect our results below, as we derive a value for $\beta$ relatively close to unity. A star approaching the $\mathrm{BH}$ is considered to be on a parabolic orbit, that is, $\beta \leqslant 1$. Assuming the most conservative value for the penetration factor $\beta=1$, a mass for the main sequence star in the range $0.1-100 M_{\odot}$, a spin value in the range of $(1 ; 3)$, and $\log \left(M_{\mathrm{BH}} / M_{\odot}\right)=5.5 \pm 0.5$, then $\left(t_{0}-t_{\mathrm{D}}\right) \in(0.002 ; 0.19) \mathrm{yr}$, corresponding to $(0.8 ; 70)$ days.

Our light curve analysis shows a steeper decay than typically expected for a TDE case (the canonical $n=5 / 3$ power-law). Initially, Lodato \& Rossi (2011) proposed a more rapid decay for the X-ray light curve at late times due to a change in the spectral behavior caused by a drop in the BB temperature. Our $\mathrm{X}$-ray spectral analysis suggests no such spectral evolution. A different possibility is that the light curve shape is affected by the dynamic of the encounter (Cannizzo et al. 2011; Guillochon \& Ramirez-Ruiz 2013). According to the hydrodynamical simulations of Guillochon \& Ramirez-Ruiz (2013), the peak will occur at

$$
t_{\text {peak }}=\left(t_{0}-t_{\mathrm{D}}\right)=B_{\gamma} M_{6}^{1 / 2} r_{*}^{3 / 2} m_{*}^{-1} \mathrm{yr},
$$

while the asymptotic light curve slope decay is

$$
n_{\infty}=D_{\gamma},
$$

where $B_{\gamma}$ and $D_{\gamma}$ are functions of the penetration factor $\beta$, with different behavior if the polytropic index $\gamma$ is $4 / 3$ or $5 / 3$, values assumed for high- and low-mass main-sequence stars, respectively. From the light curve analysis $n=n_{\infty}=2.44 \pm$ 0.40 , this means that $\beta$ must be in the range $0.6-0.8$ if $\gamma=5 / 3$ and $0.7-1.7$ if $\gamma=4 / 3$. Consequently, $\left(t_{0}-t_{\mathrm{D}}\right) \in(19 ; 72)$ and $(9 ; 66)$ days for the two stellar structures, respectively. The latest interval can be further shortened by considering the fastest decay in Guillochon \& Ramirez-Ruiz (2013): their steeper decay $n_{\infty} \sim 2.2$ is obtained with $\gamma=4 / 3$ and $\beta \in(0.9 ; 1.6)$, corresponding to $\left(t_{0}-t_{\mathrm{D}}\right) \in(16 ; 25)$ days. A much shorter timescale is obtained assuming the canonical $n=5 / 3$ decay, an average $\mathrm{BH}$ mass of $10^{5.5} M_{\odot}$ and a solartype $\operatorname{star}\left(m_{*}=r_{*}=1\right)$ : we find $\left(t_{0}-t_{\mathrm{D}}\right)=0.012 \mathrm{yr}$ (i.e., 4 days).

Since $t_{\mathrm{D}}=1997.957$, we find that $t_{0}$ very likely happened before the observation by EUVE (1998.236) both using the canonical or the new theoretical assumptions, confirming the results from the light curve analysis.

\subsection{Energetics}

The analysis of the X-ray spectrum indicates that only thermal emission is necessary. The most obvious interpretation is that we are seeing emission from an accretion disk generated by a TDE. Assuming a thermal, BB model with $k T=0.09 \mathrm{keV}$ as the best description of the X-ray spectrum over the duration of the flare, the factor to convert the $0.5-7 \mathrm{keV}$ into bolometric luminosity is 10.5. The conversion factor might be slightly higher if the X-ray spectrum is more complex, as possibly seen in the first Chandra observation in 1999. Despite the good fit obtained with a powerlaw in that observation, we do not think that this is the correct description of the photon SED: by extrapolating the unabsorbed flux from Chandra into the EUVE energy range at the time of the EUVE observation in 1998 using the power-law model we found an absorbed, observed flux of $1.6 \times 10^{-11} \mathrm{erg} \mathrm{cm}^{-2} \mathrm{~s}^{-1}$, which is $\sim 4$ times what we measured.

We estimate the total released energy by integrating the light curve over the course of the flare. Lodato \& Rossi (2011) showed that the bolometric luminosity light curve for a $10^{6} M_{\odot} \mathrm{BH}$ and solar-type star is compatible with the typical decay $(n=5 / 3)$ only $\sim 200$ days after the event, while it departs from that decay at earlier times: at the time of the peak Lodato \& Rossi (2011) predict a bolometric luminosity $\sim 4$ times lower than if the $5 / 3$ decay is assumed. We started integrating the light curve at the earliest point $\left(t_{0}-t_{\mathrm{D}}\right)=0.012 \mathrm{yr}$ (see above) and we stopped at the last detection by Chandra (on 2004 January 18). We found a value for the total released energy of $E=1.7 \times 10^{52} \mathrm{erg}$ at the cluster redshift. Assuming a standard mass-to-energy conversion factor $\epsilon=0.1$, the mass accreted over the $6 \mathrm{yr}$ time frame is then $M_{\text {acc }}=E /\left(\epsilon c^{2}\right) \sim 0.10 M_{\odot}$. Ayal et al. (2000) showed through numerical simulations that for a $\mathrm{BH}$ with $10^{6} M_{\odot}$ mass and a solar-type disrupted star, only $\sim 10 \%$ of the star is accreted, i.e., the assumption of a solar-type star in our case is consistent with these results. 
The bound mass forms a disk and accretes on the BH initially at a high rate. Rees (1988) and Phinney (1989) showed that the mass accretion rate is

$$
\dot{M}_{\text {fallback }} \approx \frac{1}{3} \frac{m_{*}}{t_{\text {fallback }}}\left(\frac{t}{t_{\text {fallback }}}\right)^{-5 / 3} .
$$

At the fallback time (4 days), the accretion rate was $\sim 28 M_{\odot} \mathrm{yr}^{-1}$ but it fell quickly and at the time of the EUVE observation $(\sim 100$ days $)$, the rate was $\sim 0.1 M_{\odot} \mathrm{yr}^{-1}$. For a $10^{5.5} M_{\odot}$ BH mass, the Eddington accretion rate is $\dot{M}_{\mathrm{Edd}} \equiv$ $10 L_{\text {Edd }} / c^{2}=7.0 \times 10^{-3} M_{\odot} \mathrm{yr}^{-1}$, where $L_{\text {Edd }}$ is the Eddington luminosity $\left(4.1 \times 10^{43} \mathrm{erg} \mathrm{s}^{-1}\right)$, and 0.1 is the efficiency. This means that up to the EUVE observation, the accretion rate was still super-Eddington. During this phase the formed disk is thought to be geometrically thick, optically thin and highly advective (King \& Pounds 2003). The change from super- to subEddington rate happens at $t_{\mathrm{Edd}} \sim 0.1 M_{6}^{2 / 5} R_{\mathrm{p}, 3 \mathrm{R}_{\mathrm{s}}}^{6 / 5} m_{*}^{3 / 5} r_{*}^{-3 / 5} \mathrm{yr}$ (Strubbe \& Quataert 2009), where $R_{\mathrm{p}, 3 \mathrm{R}_{\mathrm{S}}}$ is the pericenter distance in units of 3 Schwarzschild radii, $R_{\mathrm{S}}$. Assuming $M_{\mathrm{BH}}=10^{5.5} M_{\odot}$, a solar-type star, and $t_{\mathrm{Edd}} \sim 1.7 \mathrm{yr}$ (from Equation (4)), this implies that $R_{\mathrm{p}, 3 \mathrm{R}_{\mathrm{S}}} \sim 14.7$, or $\sim 4.1 \times 10^{12} \mathrm{~cm}$. Since $R_{\mathrm{t}}=r_{*} m_{*}^{-1 / 3} M_{\mathrm{BH}}^{1 / 3}=4.8 \times 10^{12} \mathrm{~cm}$, the penetration factor is $\beta \sim 1.2$.

The results are only slightly different if the model of Guillochon \& Ramirez-Ruiz (2013) and the assumptions explained above $\left(M_{\mathrm{BH}}=10^{5.5} M_{\odot}, \gamma=4 / 3\right.$ and $\left.\beta \in(0.9 ; 1.6)\right)$ are used: The accretion rate at the time of the peak (between 16 and 25 days) varies from 0.36 to $4.8 M_{\odot} \mathrm{yr}^{-1}$, while it becomes sub-Eddington at sometimes between 2.9 and $3.5 \mathrm{yr}$ after the disruption.

Li et al. (2002) showed that the accretion disk has a characteristic radius $\left(R_{\mathrm{X}}\right)$ that might be estimated from the X-ray spectral analysis. By requiring a $\mathrm{BB}$ model with temperature $T_{\mathrm{bb}}$ and assuming a correction factor of $f_{\mathrm{c}} \geqslant 1$ (Ross et al. 1992) to compensate for spectral hardening by Comptonization and electron scattering, the radius can be expressed as

$$
R_{\mathrm{X}}=\left(\frac{L_{\mathrm{bol}} f_{\mathrm{c}}^{4}}{\pi \sigma T_{\mathrm{bb}}^{4}}\right)^{1 / 2},
$$

where $L_{\mathrm{bol}}$ is the bolometric luminosity and $\sigma$ is the Stefan-Boltzmann constant. Since our Chandra spectra were fit with a BB model with $T_{\mathrm{bb}}=10^{6} \mathrm{~K}$, and at the time of the $E U V E$ observation $L_{\text {bol }}$ was $3.3 \times 10^{44} \mathrm{erg} \mathrm{s}^{-1}$, we find that $R_{\mathrm{X}}$ is $\geqslant 1.36 \times 10^{12} \mathrm{~cm}$. This radius coincides with the tidal radius if a correction factor $f_{\mathrm{c}} \sim 2$ is assumed, a value similar to what has been found appropriate for these conditions (i.e., $f_{\mathrm{c}}=3$; Shimura \& Takahara 1993; Li et al. 2002). $R_{\mathrm{X}}$ is where the inner edge of the debris stream and the corresponding elliptical disk should be located, assuming that the debris stream is centered at $R \sim 2 \times R_{\mathrm{p}}$. The radius does not correspond to the innermost stable circular orbit $\left(R_{\mathrm{ISCO}}\right)$ that is expected to mark the inner edge of the accretion disk. Since $R_{\mathrm{ISCO}}=3 \times R_{\mathrm{S}}$ for a nonspinning $\mathrm{BH}$, it is at least an order of magnitude smaller than the estimated $R_{\mathrm{X}}$.

\section{SUMMARY}

We serendipitously discovered a high energy transient in the field of view of the moderately rich cluster A1795. The flare was discovered in observations with the EUVE and Chandra satellites: the first detection was on 1998 March 27 in the UV and the last glimpse was on 2004 January 18 in the X-rays.
Previous observations by EUVE and ROSAT up to 1997 July do not reveal any emission at the transient position. A total of seven observations are used to generate the X-ray light curve: the brightness of CXO J1348 decays as $a \times\left[\left(t-t_{\mathrm{D}}\right) /\left(t_{0}-t_{\mathrm{D}}\right)\right]^{-n}$, where $n=2.44 \pm 0.40$, a behavior seen in previous cases of TDE candidates (Cappelluti et al. 2009) and in agreement with recent hydrodynamical simulations (Guillochon \& RamirezRuiz 2013). The start of this event $t_{D}$ can be set a few weeks before the first observation. The spectral analysis of the Chandra data are consistent with this interpretation. There is not significant emission in the hard X-rays (above 2-3 keV) associated with this event, and the fit of the soft spectrum can be obtained using a thermal model. The lack of any flat powerlaw component rules out some of the candidate progenitors, such as AGNs and GRBs. Also a supernova explosion is excluded because of the very short-term variability observed by Chandra over a four-day time range, as well as the lack of star formation in the host. Throughout the first $3 \mathrm{yr}$ of Chandra observations the temperature of the $\mathrm{BB}$ model is constant around $k T=0.09 \mathrm{keV}$, corresponding to $T=10^{6} \mathrm{~K}$. Assuming this model to fit the EUVE observation as well, we estimated that the $0.5-7 \mathrm{keV}$ unabsorbed flux changes by a factor of $\sim 2300$, a value higher than numerous other TDEs and consistent with the most extreme cases monitored over very long periods (e.g., Chandra observations of RX J1624.9+7554 showed a decline by a factor of 6000 from its ROSAT peak; Halpern et al. 2004).

At the position of CXO J1348 we found a host galaxy using ground- and space-based telescopes. Combining archival with new and deeper observations, we were able to estimate a photo-redshift. The best fit of the SED can be obtained with an elliptical/S0 galaxy located in proximity of A1795. The estimated absolute magnitude place this galaxy at the very bottom of previously studies of galaxies containing either an SMBH or a compact nucleus. Using different methods (Graham \& Scott 2013; Kormendy \& Ho 2013) we concluded that the BH in the center of the host might not be supermassive $\left(10^{5.5 \pm 0.5} M_{\odot}\right)$. Only a handful of cases has been reported so far in which an IMBH has been found in the host galaxy (see Section 1). Wang $\&$ Merritt (2004) predicted that disruptions happen with a rate of $\sim 10^{-4}-10^{-5}$ galaxy $^{-1} \mathrm{yr}^{-1}$ for galaxies with SMBH, while the rate is highest in nucleated dwarf galaxies, reaching a value as high as $\sim 10^{-3}$ galaxy $^{-1} \mathrm{yr}^{-1}$ in faint nucleated spheroids. During the course of $12 \mathrm{yr}$ of observations of A1795, Chandra covered extensively the cluster inner part ( $10^{\prime}$ in radius). In that area there are more than 2000 objects that have an optical magnitude between 21 and 24, a plausible range for dwarf spheroids at the cluster distance. Assuming that only $10 \%$ of those objects are located at that distance, than the measured rate is also in the range $\sim 10^{-3}-10^{-4}$. Thus, our serendipitous discovery can be explained by this high disruption rate and argues in favor the existence of an IMBH in a dwarf galaxy.

The host shows no significant variability over a very wide time range (from 1999 to 2013), while an optical spectrum obtained with the LBT $15 \mathrm{yr}$ after the event does not show the presence of emission lines, supporting the idea that the optical emission is dominated by the underlying quiescent galaxy. Given the high temperature observed in the X-ray spectrum, the optical transient would not be observed, even at the time of the X-ray peak: the estimated flux at $5500 \AA$ assuming a simple BB model would have been $\sim 0.02 \mu \mathrm{Jy}$, corresponding to $\operatorname{mag}_{V}=$ 28. It has been shown (Strubbe \& Quataert 2009; Lodato \& Rossi 2011) that the optical radiation might be dominated by emission generated in wind outflows during the super-Eddington 
phase. This enhancement can be up to two orders of magnitudes, corresponding to $\operatorname{mag}_{V}=23$, similar to the value of the entire host galaxy. Only with observations performed within a few months after the event it would have been possible to detect this increase. Unfortunately, the first available observation, obtained by $H S T$, happened more than a year after the disruption and no enhancement has been observed.

Almost all the known TDE cases are not detected in the radio bands and have a $\mathrm{X}$-ray luminosity of $L_{\mathrm{X}} \lesssim 10^{45} \mathrm{erg} \mathrm{s}^{-1}$ (Komossa 2002). The only exceptions are Sw J1644+57 and Sw J2058+05, whose detection at low frequencies and large luminosity in the X-rays $\left(L_{\mathrm{X}}>10^{47} \mathrm{erg} \mathrm{s}^{-1}\right.$, Cannizzo et al. 2011; Burrows et al. 2011) suggest the presence of a jet of relativistic, collimated plasma which produces synchrotron emission and boosts the luminosity by beaming. In our case the extrapolated bolometric luminosity is $2.8 \times 10^{48} \mathrm{erg} \mathrm{s}^{-1}$ at the time of the theoretical peak, 2.4 days after the event. Lodato \& Rossi (2011) showed that this is likely a very conservative upper limit because the light curve is flatter in the first part of the system evolution. The lack of any radio signal in any observations from 2000 to 2005 indicates that no jet has been created or that the direction of the collimated plasma does not coincide with the line of sight. It has been proposed and shown (e.g., Giannios \& Metzger 2011; van Velzen et al. 2011; Bower et al. 2013) that radio emission can be visible one or more years after the TDE, when the plasma decelerates to mildly relativistic speed due to interaction with the interstellar medium or when the jet becomes radio-loud as a function of the accretion rate. New radio observations are needed to check if this assumption applies to this case.

This study highlights, once again, the importance of an $\mathrm{X}$-ray monitoring campaign of clusters of galaxies to discover and better characterize this kind of event. As shown above, the lack of information in the very early part of the event prevented us to put firm constraints to the system. Future monitoring programs in the X-rays, such as eROSITA (e.g., Predehl et al. 2007), would be extremely important to find other TDEs associated with IMBHs, whose existence is still open to debate.

We thank the anonymous referee for constructive comments, which helped us to improve the manuscript.

Observations have been carried out using the Large Binocular Telescope (LBT) at Mt. Graham, AZ., the Johnson Telescope at the Observatorio Astronomico Nacional/San Pedro Mártir in Mexico, and the Nordic Optical Telescope at La Palma, Spain.

The LBT is an international collaboration among institutions in the United States, Italy, and Germany. LBT Corporation partners are The University of Arizona on behalf of the Arizona university system; Istituto Nazionale di Astrofisica, Italy; LBT Beteiligungsgesellschaft, Germany, representing the MaxPlanck Society, the Astrophysical Institute Potsdam, and Heidelberg University; The Ohio State University; and The Research Corporation, on behalf of The University of Notre Dame, University of Minnesota, and University of Virginia. This paper used data obtained with the MODS spectrographs built with funding from NSF grant AST-9987045 and the NSF Telescope System Instrumentation Program (TSIP), with additional funds from the Ohio Board of Regents and the Ohio State University Office of Research.

We thank the staff of the Observatorio Astronómico Nacional on Sierra San Pedro Mártir and the RATIR instrument team (ratir.astroscu.unam.mx). RATIR observations with the Harold
L. Johnson $1.5 \mathrm{~m}$ telescope of the Obervatorio Astronómico Nacional on Sierra San Pedro Mártir are partially funded by UNAM, CONACyT and NASA, and supported by the loan of an H2RG detector by Teledyne Scientific and Imaging.

The data presented here were obtained in part with ALFOSC, which is provided by the Instituto de Astrofisica de Andalucia (IAA) under a joint agreement with the University of Copenhagen and NOTSA.

C.C.C. was supported at NRL by a Karles' Fellowship and NASA DPR S-15633-Y.

H.L. acknowledges financial support by the European Union through the COFUND scheme.

We thank Marco Fumana from the LBT data center for quickly providing the reduced observations; Martin Sirk for helping in the EUVE analysis; Dan Perley for estimating the photo-redshift; and Sjoert van Velzen for providing additional comments on the discussion.

Facilities: EUVE(DS), HST (WFPC2), CXO (ACIS), ROSAT (PSPC), VLA, VLT (FORS1), LBT (MODS), OANSPM:HJT (RATIR), NOT

\section{REFERENCES}

Ahn, C. P., Alexandroff, R., Allende Prieto, C., et al. 2012, ApJS, 203, 21 Ayal, S., Livio, M., \& Piran, T. 2000, ApJ, 545, 772

Bade, N., Komossa, S., \& Dahlem, M. 1996, A\&A, 309, L35 Bloom, J. S., Giannios, D., Metzger, B. D., et al. 2011, Sci, 333, 203 Bloom, J. S., Kulkarni, S. R., \& Djorgovski, S. G. 2002, AJ, 123, 1111 Bonamente, M., Lieu, R., \& Mittaz, J. P. D. 2001, ApJL, 547, L7 Boulade, O., Charlot, X., Abbon, P., et al. 2003, Proc. SPIE, 4841, 72 Bower, G. C., Metzger, B. D., Cenko, S. B., Silverman, J. M., \& Bloom, J. S. 2013, ApJ, 763, 84

Bowyer, S., Berghöfer, T. W., \& Korpela, E. J. 1999, ApJ, 526, 592 Bowyer, S., \& Malina, R. F. 1991, ASR, 11, 205

Brammer, G. B., van Dokkum, P. G., \& Coppi, P. 2008, ApJ, 686, 1503 Brandt, W. N., Mathur, S., \& Elvis, M. 1997, MNRAS, 285, L25 Bruzual, G., \& Charlot, S. 2003, MNRAS, 344, 1000 Burrows, D. N., Kennea, J. A., Ghisellini, G., et al. 2011, Natur, 476, 421 Butler, N. R. 2007, ApJ, 656, 1001

Butler, N., Klein, C., Fox, O., et al. 2012, Proc. SPIE, 8446, 10 Cannizzo, J. K., Troja, E., \& Lodato, G. 2011, ApJ, 742, 32 Cappelluti, N., Ajello, M., Rebusco, P., et al. 2009, A\&A, 495, L9 Cardelli, J. A., Clayton, G. C., \& Mathis, J. S. 1989, ApJ, 345, 245

Cenko, S. B., Bloom, J. S., Kulkarni, S. R., et al. 2012, MNRAS, 420, 2684 Cenko, S. B., Krimm, H. A., Horesh, A., et al. 2012, ApJ, 753, 77

Christian, D. J., Craig, N., Cahill, W., Roberts, B., \& Malina, R. F. 1999, AJ, 117,2466

Chornock, R., Berger, E., Gezari, S., et al. 2013, arXiv:1309.3009 D’Avanzo, P., Salvaterra, R., Sbarufatti, B., et al. 2012, MNRAS, 425, 506 Donato, D., Ghisellini, G., Tagliaferri, G., \& Fossati, G. 2001, A\&A, 375, 739 Dong, R., Greene, J. E., \& Ho, L. C. 2012, ApJ, 761, 73

Donley, J. L., Brandt, W. N., Eracleous, M., \& Boller, T. 2002, AJ, 124, 1308

Dwarkadas, V. V., \& Gruszko, J. 2012, MNRAS, 419, 1515

Esquej, P., Read, A. M., \& Saxton, R. D. 2010, in AIP Conf. Proc. 1248, X-ray Astronomy 2009, Present Status, Multi-Wavelength Approach and Future Perspectives, ed. A. Comastri, L. Anjelini, \& M. Cappi (Melville, NY: AIP), 237

Esquej, P., Saxton, R. D., Freyberg, M. J., et al. 2007, A\&A, 462L, 49

Esquej, P., Saxton, R. D., Komossa, S., et al. 2008, A\&A, 489, 543

Evans, C. R., \& Kochanek, C. S. 1989, ApJL, 346, L13

Fazio, G. G., Hora, J. L., Allen, L. E., et al. 2004, ApJS, 154, 10

Fox, O. D., Kutyrev, A. S., Rapchun, D. A., et al. 2012, Proc. SPIE, 8453, 10 Frank, J., \& Rees, M. J. 1976, MNRAS, 176, 633

Fruchter, A. S., Levan, A. J., Strolger, L., et al. 2006, Natur, 441, 463 Ge, J. P., \& Owen, F. N. 1993, AJ, 105, 778

Gezari, S., Basa, S., Martin, D. C., et al. 2008, ApJ, 676, 944

Gezari, S., Chornock, R., Rest, A., et al. 2012, Natur, 485, 217

Gezari, S., Heckman, T., Cenko, S. B., et al. 2009, ApJ, 698, 1367

Gezari, S., Martin, D. C., Forster, K., et al. 2013, ApJ, 766, 60

Gezari, S., Martin, D. C., Milliard, B., et al. 2006, ApJL, 653, L25

Giannios, D., \& Metzger, B. D. 2011, MNRAS, 416, 2102

Giommi, P., Polenta, G., Lähteenmäki, A., et al. 2012, A\&A, 541, A160 
Graham, A. W., \& Scott, N. 2013, ApJ, 764, 151

Greene, J. E., \& Ho, L. C. 2007, ApJ, 670, 92

Greiner, J., Schwarz, R., Zharikov, S., \& Orio, M. 2000, A\&A, 362, L25

Grupe, D., Beuermann, K., Mannheim, K., et al. 1995, A\&A, 299, L5

Grupe, D., Komossa, S., Gallo, L. C., et al. 2012, ApJS, 199, 28

Guillochon, J., \& Ramirez-Ruiz, E. 2013, ApJ, 767, 25

Halpern, J. P., Gezari, S., \& Komossa, S. 2004, ApJ, 604, 572

Hill, J. M., \& Oegerle, W. R. 1993, AJ, 106, 831

Hill, J. M., \& Salinari, P. 2000, Proc. SPIE, 4004, 36

Holtzman, J. A., Burrows, C. J., Casertano, S., et al. 1995, PASP, 107, 1065

Hopkins, P. F., \& Hernquist, L. 2006, ApJS, 166, 1

Irwin, J. A., Brink, T. G., Bregman, J. N., \& Roberts, T. P. 2010, ApJ, 712, L1

Kalberla, P. M. W., Burton, W. B., Hartmann, D., et al. 2005, A\&A, 440, 775

Kanner, J., Baker, J., Blackburn, L., et al. 2013, ApJ, 774, 63

Karttunen, H. (ed.) 1993, NOT (Nordic Optical Telescope) user manual, version 1, 1992, Obs. Rep., Informo, No. 170

King, A. R., \& Pounds, K. A. 2003, MNRAS, 345, 657

Komossa, S. 2002, RvMA, 15, 27

Komossa, S., \& Greiner, J. 1999, A\&A, 349, L45

Komossa, S., Halpern, J., Schartel, N., et al. 2004, ApJL, 603, L17

Komossa, S., Zhou, H., Wang, T., et al. 2008, ApJL, 678, L13

Kormendy, J., \& Ho, L. C. 2013, ARA\&A, 51, 511

Kormendy, J., \& Richstone, D. 1995, ARA\&A, 33, 581

Krist, J. E., Hook, R. N., \& Stoehr, F. 2011, Proc. SPIE, 8127, 16

Krolik, J., \& Piran, T. 2011, ApJ, 743, 134

Leighly, K. M. 1999, ApJS, 125, 317

Levan, A. J., Tanvir, N. R., Cenko, S. B., et al. 2011, Sci, 333, 199

Lewis, J. R., Bunclark, P. S., Irwin, M. J., McMahon, R. G., \& Walton, N. A. 2000, in ASP Conf. Ser. 216, Astronomical Data Analysis Software and Systems IX, ed. N. Manset, C. Veillet, \& D. Crabtree (San Francisco, CA: ASP), 415

Li, L.-X., Narayan, R., \& Menou, K. 2002, ApJ, 576, 753

Lin, D., Carrasco, E. R., Grupe, D., et al. 2011, ApJ, 738, 52

Lodato, G., King, A. R., \& Pringle, J. E. 2009, MNRAS, 392, 332

Lodato, G., \& Rossi, E. M. 2011, MNRAS, 410, 359

Lu, Y., Huang, Y. F., \& Zhang, S. N. 2008, ApJ, 684, 1330

Maksym, W. P., Ulmer, M. P., \& Eracleous, M. 2010, ApJ, 722, 1035

Maksym, W. P., Ulmer, M. P., Eracleous, M. C., Guennou, L., \& Ho, L. C. 2013, MNRAS, 435, 1904

McGee, S. L. 2013, MNRAS, 436, 2708
McHardy, I. M., Papadakis, I. E., Uttley, P., Page, M. J., \& Mason, K. O. 2004, MNRAS, 348, 783

Miniutti, G., Ponti, G., Greene, J. E., et al. 2009, MNRAS, 394, 443

Nicklas, H., Seifert, W., Boehnhardt, H., Kiesewetter-Koebinger, S., \& Rupprecht, G. 1997, Proc. SPIE, 2871, 1222

Nikołajuk, M., \& Walter, R. 2013, A\&A, 552, A75

Oegerle, W. R., \& Hill, J. M. 2001, AJ, 122, 2858

Perley, D. A., Modjaz, M., Morgan, A. N., et al. 2012, ApJ, 758, 122

Phinney, E. S. 1989, Natur, 340, 595

Pian, E., Romano, P., Maoz, D., et al. 2010, MNRAS, 401, 677

Porquet, D., Reeves, J. N., O’Brien, P., \& Brinkmann, W. 2004, A\&A, 422, 85

Predehl, P., Andritschke, R., Bornemann, W., et al. 2007, Proc. SPIE, 6686, 36

Rees, M. J. 1988, Natur, 333, 523

Richards, E. A., Kellermann, K. I., Fomalont, E. B., Windhorst, R. A., \& Partridge, R. B. 1998, AJ, 116, 1039

Roming, P. W. A., Kennedy, T. E., Mason, K. O., et al. 2005, SSRv, 120, 95

Ross, R. R., Fabian, A. C., \& Mineshige, S. 1992, MNRAS, 258, 189

Savaglio, S., Glazebrook, K., \& Le Borgne, D. 2009, ApJ, 691, 182

Saxton, R. D., Read, A. M., Esquej, P., et al. 2008, A\&A, 480, 611

Saxton, R. D., Read, A. M., Esquej, P., et al. 2012, A\&A, 541, A106

Schlafly, E. F., \& Finkbeiner, D. P. 2011, ApJ, 737, 103

Shcherbakov, R. V., Penna, R. F., \& McKinney, J. C. 2012, ApJ, 755, 133

Shepherd, M. C., Pearson, T. J., \& Taylor, G. B. 1994, BAAS, 26, 987

Shimura, T., \& Takahara, F. 1993, ApJ, 419, 78

Skrutskie, M. F., Cutri, R. M., Stiening, R., et al. 2006, AJ, 131, 1163

Smith, R. J., Hudson, M. J., Nelan, J. E., et al. 2004, AJ, 128, 1558

Spergel, D. N., Verde, L., Peiris, H. V., et al. 2003, ApJS, 148, 175

Stone, N., Sari, R., \& Loeb, A. 2013, MNRAS, 435, 1809

Strubbe, L. E., \& Quataert, E. 2009, MNRAS, 400, 2070

Strubbe, L. E., \& Quataert, E. 2011, MNRAS, 415, 168

Ulmer, A. 1999, ApJ, 514, 180

Urry, C. M., \& Padovani, P. 1995, PASP, 107, 803

Urry, C. M., Scarpa, R., O’Dowd, M., et al. 2000, ApJ, 532, 816

van Velzen, S., Farrar, G. R., Gezari, S., et al. 2011, ApJ, 741, 73

Varela, J., D’Onofrio, M., Marmo, C., et al. 2009, A\&A, 497, 667

Wang, J., \& Merritt, D. 2004, ApJ, 600, 149

Watson, A. M., Richer, M. G., Bloom, J. S., et al. 2012, Proc. SPIE, 8444, 5L

Weisskopf, M. C., Hester, J. J., Tennant, A. F., et al. 2000, ApJL, 536, L81

Wright, E. L. 2006, PASP, 118, 1711

Zauderer, B. A., Berger, E., Soderberg, A. M., et al. 2011, Natur, 476, 425

Zhou, H., Wang, T., Yuan, W., et al. 2006, ApJS, 166, 128 